PEP-II AP Note 18-93

\title{
Ion Trapping in the SLAC B-factory High Energy Ring
}

\author{
D. Villevald and S. Heifets
}


PEP-I1 AP Note 18-9.3

June 17, 1993

\section{Ion trapping \\ in the SLAC B-factory High Energy Ring. \\ D. Villevald, S. Heifets \\ Stanford Linear Accelerator Center \\ Stanford, California, USA}

May 1993

\section{Contents}

1. Introduction.

2. Ion production.

3. Ion trapping in the train of bunches.

4. Transverse stability of ions.

5.1 Linear motion

5.2 Non-linear motion

5.3 Distribution function of ions

5. The longitudinal stability.

6. Effects of the accumulated ions.

6.1 One-turn ions

6.2 Unstable ions

6.3 Trapped ions

7. Conclusion and some comments.

8. Acknowledgments.

A. The electromagnetic field of an electron bunch.

B. Simulation of the nonlinear motion of ions. 


\section{Introduction.}

The presence of trapped ions in electron storage rings has caused significant degradation in machine performance. The best known way to prevent the ion trapping is to leave a gap in the electron bunch train. The topic of this paper is the dynamics of ions in the field of the bunch train with uneven bunch filling. We consider High Energy Ring (HER) of the PEP-I1 B-factory. The relevant parameters of the electron ring are:

revolution time $T=7.3 \mu \mathrm{sec}$,

energy $E$, $=9.0 \mathrm{GeV}$,

dc beam current $I_{0}=0.99 \mathrm{~A}$,

number of bunches $\mathbf{n g}=1658$,

number of particles per bunch $N_{B}=2.7510^{10}$,

the rms emittances $\epsilon_{x}=48.2 \mathrm{~nm}$ and $\epsilon_{y}=\mathbf{1 . 9 3} \mathrm{nm}$,

rms energy spread $\delta=6.110^{-4}$,

lattice functions of the $60^{\circ}$ lattice: $\beta_{\max }=25 \mathrm{~m}, \beta_{\min } / \beta_{\max }=1 / 3, \eta_{\max }=12 \mathrm{~m}$, $\eta_{\max } / \eta_{\min }=5 / 3$

the bunch spacing is $4.2 \mathrm{nsec}$ and the bunch length is $1 \mathrm{~cm}$.

In the first section we summarize mechanisms of the ion production. Then the transverse and longitudinal dynamics are analysed for a beam with and without gap. After that, the effect of the ions is considered separating all ions in the ring in several groups depending on their transverse and longitudinal stability. The main effects of the ions are the tune shift and the tune spread of the betatron oscillations of the electrons. The tune spread is produced by bunch to bunch variation of the electric field of ions and by nonlinearity of the field. It is shown that the main contribution to the shift and spread of the betatron tune of the beam is caused by two groups of ions: one-turn ions and trapped ions.

One-turn ions are the ions generated during the last passage of the bunch train. Their effect depends on the distribution function of the ions. The distribution function, found by Tavares ${ }^{[15]}$, is much narrower than the beam rms size. That enchances effect of ions on the beam giving the bunch-to-bunch betatron tune spread $\Delta \nu=0.02$ for the design current $I=0.99 \mathrm{~A}$ in the High Energy Ring.

Trapped ions are the ions with stable transverse and longitudinal motion. It is shown that the betatron tune shift caused by all trapped ions is of the order of the tune spread due to the beam-beam interaction, namely $\Delta \nu=0.03$. This estimate, however, is conservative and obtained for the worst situation when stability zones are located at the position of the defocusing quads and all quads in the ring are identical. Effect can be decreased by the factor of two by increasing the length of the gap from $5 \%$ to $10 \%$. It is worth noting, that the interaction of an electron with ions has higher repetition rate than the beam-beam interaction and, therefore, the effect of the ions is not necessarily the same as the effect of the beam-beam collisions.

Because the effect of trapped ions comparable to the effect of one-turn ions, we come to the conclusion that clearing electrodes are not necessary. However, they can be introduced later. Passive matched lines, which can also be usefull for damping the transverse collective oscillations, may be used for this purpose. 
In the last section we discuss shortly related problems of parameters of the clearing electrodes, injection scenario, and collective effects. Clearing electrodes should be located at the defocusing in x-plane quadrupole magnets. An electric DC field of value $1.0 \frac{\mathrm{kV}}{\mathrm{cm}}$ will be enough to prevent the ion trapping process. During the injection, it is recommended to fill the bucket with the design number of the particles per bunch $N_{B}$ before going to the next bucket. In addition, it is recommended to have the sequential filling of the ring, i.e. the filling from one bucket to the next sequentially. It was shown that ions will not be trapped at the location of the interaction point. The reason for this is that the current of the positron beam is twice as large as the current of the electron beam, while the transverse sizes of both the electron and positron beams are equal at the IP. It is shown that the linear ion's oscillations can not result in the longitudinal coupled beam instabilities.

\section{Ion production.}

Ions can be generated in the inelastic collisions of the circulating electron beam with the molecules of residual gas, by tunneling ionization due to the collective electric field of the bunch, in the Compton scattering of the synchrotron radiation on the electrons of residual gas molecules, and the ion desorption from the wall.

The first mechanism of the ion production is the main one. The cross section of the ionization may be parametrized in the form

$$
\sigma_{c o l}=4 \pi \lambda_{c}^{2} \beta^{2}\left(C+2 M^{2}\left(\ln \beta \gamma-\frac{1}{2}\right)\right)
$$

where $C$ and $M^{2}$ depend upon the gas ${ }^{[1]}, \lambda_{c}=\hbar / m_{e} c$ is the Compton wavelength of electron, $\gamma$ is the relativistic factor, and $\mathrm{c}$ is the speed of light.

The residual gas in the High Energy Ring vacuum chamber is composed of molecular hydrogen $(75 \%)$, carbon monoxide (14\%), carbon dioxide $(7 \%)$, methane (less than $4 \%$ ) and small amount of other molecular species.

Table $\mathbf{1}$ shows atomic number $\mathbf{A}$, cross sections $\sigma_{\text {coll }}$, partial densities $\mathbf{n}$, and ionization times $\tau_{c o l}=\left(n c \sigma_{c o l}\right)^{-1}$ for the main molecular species in the HER vacuum chamber for the average pressure $p=5 \mathrm{n}$ Torr and temperature $T=300 \mathrm{~K}^{\circ}$.

Table 1. The parameters of collision ionization for the main molecular species at the HER.

\begin{tabular}{|c|c|c|c|c|}
\hline & A & $\sigma_{\text {col }}, \mathrm{cm}^{2}$ & $\tau_{c o l}, \mathrm{sec}$ & $n, \frac{1}{c m^{3}}$ \\
\hline$H_{2}$ & 2 & $3.510^{-19}$ & 0.77 & $1.210^{8}$ \\
\hline$C O$ & 28 & $1.910^{-18}$ & 0.77 & $2.310^{7}$ \\
\hline $\mathrm{CO}_{2}$ & 44 & $3.010^{-18}$ & 0.99 & $1.110^{7}$ \\
\hline $\mathrm{CH}_{4}$ & 16 & $2.110^{-18}$ & 2.59 & $6.110^{6}$ \\
\hline
\end{tabular}


Total ionization time, the time it takes for one electron in the beam to create one ion of any of the molecular species contained in the vacuum chamber, is given by

$$
\tau_{c o l}=\left(\sum_{i} \frac{1}{\tau_{c o l}^{i}}\right)^{-1}=\frac{1}{p c}\left(\sum_{i} \sigma_{c o l}^{i} \alpha_{i}\right)^{-1}=0.25 \mathrm{sec}
$$

with $\alpha_{i}=\frac{n_{i}}{n_{\text {tot }}}$ the percentage of the $\mathrm{i}$-th molecular species and $k$ the Boltzmann constant. The sum is over all molecular species.

The production rate of ions due to electron impact is

$$
\dot{N}_{c o l}=\frac{N_{e}}{\tau_{c o l}}=1.8 \cdot 10^{14} \frac{1}{\mathrm{sec}}
$$

where $N_{e}$ is the total number of electrons in the machine: $N_{e}=4.5 \cdot 10^{13}$.

The tunneling ionization can be considered as a static process since the bunch length $1 \mathrm{~cm}$ is much longer than $c \tau_{B}$ where $\tau_{B}=a_{b} /(c \alpha)$ is the typical time of electron motion in an atom and $a_{b}$ is the first Bohr radius. The transition rate for tunneling ionization in a static electric field is ${ }^{[2]}$

$$
W=8 \frac{\alpha^{3} c}{\lambda_{c}^{2}} \frac{U}{e E} \exp \left(-\frac{4}{3} \frac{\alpha}{\lambda_{c}} \frac{U}{e E}\right)\left[\sec ^{-1}\right]
$$

with $E$ is the applied electric field, $U$ the ionization energy of the order of few $\mathrm{eV}$, and or is the fine structure constant. The typical value of the collective electric field of the bunch, with length $\sigma_{s}$ and transverse rms sizes $\sigma_{x}, \sigma_{y}$, is of the order of $e E=\frac{e^{2} N_{b}}{\sigma_{s} \sigma_{y}}$, where $N_{b}$ is the number of electrons per bunch. For the HER parameters $N_{b}=2.75 \cdot 10^{10}$, $\sigma_{y}=0.02 \mathrm{~cm}$, and $\sigma_{s}=1 \mathrm{~cm}$, this gives $e E=2 \cdot 10^{5} \frac{\mathrm{eV}}{\mathrm{cm}}$. The ionization time due to the tunnelling ionization process is large compared to the ionization time (2.2) and we can neglect tunneling ionization.

Table 2 summarizes the main parameters of synchrotron radiation for the High Energy Ring ( electron ring ) ${ }^{[3]}$.

Table 2. Some characteristics of the synchrotron radiation at the HER.

\begin{tabular}{lll}
\hline Parameter & Symbol & HER \\
\hline Energy, GeV & $E_{\boldsymbol{e}}$ & 9.00 \\
Bending magnet field, T & $B$ & 0.182 \\
Magnetic radius, m & $\boldsymbol{P}$ & 165.0 \\
Power, $\mathrm{kW}$ & $\mathrm{P}$ & 5.424 \\
Critical energy in dipoles , $\mathrm{keV}$ & $\boldsymbol{\varepsilon}_{\mathrm{cr}}$ & 9.8 \\
\hline
\end{tabular}


Note here that ( see Table 2$) \varepsilon_{c r} / m_{e} c^{2}=0.02 \ll 1$. The cross-section of Compton scattering of synchrotron radiation light on the electrons of residual gas molecules. is ${ }^{(4)}$ :

$$
\sigma_{c}=\frac{8 \pi}{3} r_{e}^{2}\left(1-2 \frac{\varepsilon_{c r}}{m_{e} c^{2}}\right)=6.3 \cdot 10^{-25} \mathrm{~cm}^{2},
$$

where $r_{\boldsymbol{e}}$ is the classical radius of the electron. It is much smaller than the inelastic cross-section and this mechanism gives negligible contribution to the ion production.

Finally, the generation of ions by collisions with the electrons of the beam is predominant under the High Energy Ring parameters. The total ionization time, i.e. the time it takes for one electron to create one ion of any of the molecular species is $0.25 \mathrm{sec}$.

3. Ion trapping in the train of the equidistant electron bunches.

The ions move in the field of the electron beam. On each passage of an electron bunch, the ion gets a kick towards the beam closed orbit. To calculate this kick we can neglect the change of the ion coordinates during the time of bunch passage $\frac{\sigma_{s}}{c}$. The change of the transverse components of the ion momentum due to the bunch passage is given by

$$
\triangle p_{x}=\frac{s_{B}}{c} e E_{x}(x, y)
$$

with $e E_{x}=-\partial U_{1} / \partial x$, where $U_{1}$ is the average beam potential, see Appendix A:

$$
U_{1}=-\frac{e^{2} N_{B}}{s_{B}}\left(\int_{0}^{\infty} \frac{e^{-\frac{x^{2}}{2 \sigma_{x}^{2}+q}-\frac{y^{2}}{2 \sigma_{y}^{2}+q}}}{\sqrt{2 \sigma_{x}^{2}+q} \sqrt{2 \sigma_{y}^{2}+q}} d q-\int_{0}^{\infty} \frac{e^{-\frac{a^{2}}{2 \sigma_{x o}^{2}+q}}}{\sqrt{2 \sigma_{x o}^{2}+q} \sqrt{2 \sigma_{y o}^{2}+q}} d q\right) .
$$

The majority of the ions have been created inside the region $|x|<\sigma_{x},|y|<\sigma_{y}$. In this region the electric field of the bunch is approximately linear and is given by (A.13),(A.14). Therefore the change of the transverse components of the ion momentum due to an electron bunch passage is

$$
\triangle p_{x, y}=-\frac{e^{2} N_{B}}{c} \frac{2 x}{\sigma_{x, y}\left(\sigma_{x}+\sigma_{y}\right)}
$$

The corresponding kick of the ion velocity is given by $\Delta v_{x, y}=\frac{\Delta p_{x, y}}{m_{i}}$, where $m_{i}=\boldsymbol{A} \boldsymbol{m}$, is the mass of an ion with atomic number $A$. Because different ions are generated with equal probability along a bunch, Eq. (3.1) gives the estimate for the spread of the transverse velocities of the ions due to interaction with the bunch. Comparison of this kick with the thermal ion velocity

$$
v_{T}=\sqrt{\frac{k T}{m_{i}}}=\frac{1}{\sqrt{A}} 1.6 \cdot 105 \mathrm{sec}
$$

where $k=1.38 \cdot 10^{-16} \frac{\mathrm{erg}}{\mathrm{K}^{\circ}}$ is the Boltzman constant and $T=300 \mathrm{~K}^{\circ}$ is the temperature. shows that for the ions with $|x|<\sigma_{x},|y|<\sigma_{y}$ the thermal velocity is smaller that the 
kick (3.3):

$$
\frac{\triangle v_{x, y}^{\max }}{v_{T}} \approx \frac{20}{\sqrt{A}}
$$

We see that the motion of the ion may be considered as non-relativistic, since $\frac{\Delta v_{x, y}}{c} \sim$

It is easy to check that the change of ion coordinates for the time of the bunch passage $\frac{\sigma_{s}}{\mathrm{c}}$ is negligible small.

The Hamiltonian of the transverse motion of the single charged ion in the average field of the beam is given by

$$
H=\frac{p_{x}^{2}}{2 m_{i}}+\frac{p_{y}^{2}}{2 m_{i}}+U(x, y)
$$

For the linear region $\frac{x^{2}}{2 \sigma_{x}^{2}}, \frac{y^{2}}{2 \sigma_{y}^{2}} \ll 1$ the beam potential may be expanded in the lowest order term to the transverse coordinates. According to (A.13),(A.14) the horizontal and vertical linear ion motion are uncoupled. The one-dimensional Hamiltonian of the linear ion motion is given by

$$
H=\frac{p_{x}^{2}}{2 m_{i}}+\frac{m_{i} \Omega_{x}^{2} x^{2}}{2}+(x \rightarrow y)
$$

where $z=(x, \mathbf{y}), p_{z}=\left(p_{x}, p_{y}\right)$, and the angular frequencies of the linear ion oscillation are given by

$$
\Omega_{x, y}=\sqrt{\frac{2 N_{e} e^{2}}{C \sigma_{x, y}\left(\sigma_{x}+\sigma_{y}\right) A m_{p}}}=\sqrt{\frac{e I Z_{o}}{2 \pi A m_{p} \sigma_{x, y}\left(\sigma_{x}+\sigma_{y}\right)}}
$$

The frequencies of the order of

$$
\Omega_{y}=\frac{1}{\sqrt{A}} 1.5 \cdot 10^{8} \frac{\mathrm{rad}}{\mathrm{sec}}, \quad \Omega_{x}=\frac{1}{\mathbf{a}^{6.7} \cdot 10^{7}} \frac{\mathrm{rad}}{\mathrm{sec}}
$$

with $A$ the atomic number of the ion, $I=e c N_{e} / C=0.99 \mathrm{~A}$ the total current in the ring, $Z_{0}=377 \Omega$ the impedance of free space, $N_{e}=N_{B} n_{B}=4.5 \cdot 10^{13}$ the total number of electrons in the accelerator, $C=2.2 \cdot 10^{5} \mathrm{~cm}$ the circumference of the HER. For the estimate we used here the typical transverse rms beam sizes $\sigma_{x}=0.1 \mathrm{~cm}$, and $\sigma_{y}=0.02 \mathrm{~cm}$. The ion motion is nonlinear at large amplitudes. We may consider three typical regions: 
1) small amplitudes $x \leq \sigma_{x}, y \leq \sigma_{y}$. The first non-zero term after the parabolic one in the expansion of the Hamiltonian (4.1) near the fixed point $x=0$ y $=0$ is

$$
H \approx H_{o}-\frac{e I Z_{0}}{4 \pi} \frac{\sigma_{x} \sigma_{y}}{\left(a_{1}+\sigma_{y}\right)^{2}}\left[2 \frac{x^{2}}{2 \sigma_{x}^{2}} \frac{y^{2}}{2 \sigma_{y}^{2}}+\frac{1}{3}\left(i+2 \frac{\sigma_{x}}{\sigma_{y}}\right) \quad \frac{4}{\overline{4}}+\frac{1}{3}\left(1+2 \frac{\sigma_{y}}{\sigma_{x}}\right) \frac{{ }_{0}^{4}}{4 \sigma_{y}^{4}}\right]
$$

where $H_{0}=H,+H_{y}$, with $H_{x, y}$ the Hamiltonian of linear ion oscillations given by Eq.(3.7). It is clear that the first non-linear term in the electric field of the electron beam couples the horizontal and vertical degrees of freedom.

2)the flat beam region $\sigma_{y} \ll \mathrm{y} \ll \sigma_{x}, \mathrm{x} \ll \sigma_{x}$. For this region the integral (3.2) may be expanded to lowest order in $\frac{x^{2}}{2 \sigma_{x}^{2}}$. The result is

$$
H \approx H_{x o}+\frac{p_{y}^{2}}{2 m_{i}}+\frac{e I Z_{o}}{4 \pi} \frac{\sqrt{2}|y|}{\sigma_{x}}
$$

where $H_{x}$ is the Hamiltonian of the horizontal linear oscillations given by Eq.(3.7).

3) $x \gg$ a, and $\mathrm{y} \gg \sigma_{y}$ (the "round beam" region ).

Now we can change the lower limit of integration from 0 to $q=x^{2}+y^{2}$ and $q=a_{o}^{2}$ in the first and second terms of Eq.(3.2) respectively. Finally, the Hamiltonian of the transverse ion motion in the region of the "round beam" is

$$
H \approx \frac{p_{x}^{2}}{2 m_{i}}+\frac{p_{y}^{2}}{2 m_{i}}+\frac{e I Z_{o}}{4 \pi} \ln \left(\frac{x^{2}+y^{2}}{a_{o}^{2}}\right)
$$

Note, that ion frequencies are much larger than the revolution frequency $f_{0}=140 \mathrm{KHz}$. Therefare, all resonances related to the bunch structure

$$
k_{z} \frac{2 \pi c}{l_{b}}=2 \Omega_{z}
$$

of a train are strongly suppressed. That is true even at large amplitudes where ion frequencies are smaller than the frequencies (3.8) of small oscillations.

The depth of the potential well of the order of

$$
U\left(x=0, \mathrm{y}=0, \tilde{s}=\widetilde{s}_{o}\right)=-\frac{I Z_{0}}{2 \mathrm{x}} \ln \left(\frac{a_{o}}{\sigma_{x o}+\sigma_{y o}}\right)
$$

For the estimate we take the beam pipe radius $a_{0}=5 \mathrm{~cm}$, the total current in the ring $I=0.99 \mathrm{~A}$. For a straight sections with zero dispersion that gives $U=-\mathbf{2 4 4} \mathrm{eV}$ and $U=-223 \mathrm{eV}$ at the defocusing and focusing quads correspondingly. In the arcs potential well is shallower: $U=-143 \mathrm{eV}$ at $\mathrm{QD}$ and $U=-114 \mathrm{eV}$ at QF. The variation of the beam potential along the standard lattice cell is depicted in Fig. 1. The typical value of the depth of the potential well for the single charged ion is $\mathbf{2 2 0} \mathrm{eV}$. 


\section{Transverse stability of ions.}

\subsection{Linear motion.}

According to the previous chapters the ions of any molecular species will be linearly stable in the High Energy Ring with the equidistant bunch train operation mode. The way to avoid the ion trapping, is to leave a gap in the electron bunch train. Let us consider the conditions of the linear stability of the ion motion in this case.

The propagation of the position and momentum of the ion is given by the one-period matrix M:

$$
\mathrm{M}=\mathrm{LM}_{\mathrm{t}}
$$

with $\mathbf{L}$ the matrix of the ion drift in the gap with the length $L$, and $\mathbf{M}_{\mathrm{t}}$ the transfer matrix from the beginning to the end of the train of bunches. Introducing the revolution period of the HER, $T_{o}=7.3 \mu \mathrm{sec}$, and the $5 \%$ gap period $\mathrm{AT}=0.37 \mu \mathrm{sec}$, the instability condition of the transverse linear ion motion may be written as

$$
\left|\cos \left(\Omega_{x, y}\left(T_{o}-\Delta T\right)\right)-\frac{\Omega_{x, y} \Delta T}{2} \sin \left(\Omega_{x, y}\left(T_{o}-\Delta T\right)\right)\right|>1
$$

The phase advance for the $\boldsymbol{A}=\mathbf{4 4}$ ion's on the length of the bunch train is

$$
\Omega_{x, y}\left(T_{o}-\mathrm{AT}\right) \sim 200 \mathrm{rad}
$$

and

$$
\frac{\Omega_{x, y} \Delta T}{2} \sim 5>>1
$$

Hence, ions for all $\phi=\Omega T_{0}$ are unstable except the narrow strips $n \pi<\phi<n \pi+$ $4 /(\Delta T \Omega)$, where $n$ is an arbitrary integer. Stability corresponds to a particular combination of current, beam cross section and ion mass, such that the ion would perform nearly an exact number of half-integer oscillations during the passage of the bunch train. Fig. 2(a) shows the stability zones of the transverse motion and Fig. 2(b) the ion's oscillation frequencies $\Omega_{x}, \Omega_{y}$ as a function of the atomic number $\boldsymbol{A}$ of the ion for the HER. The frequency of the horizontal ion's oscillation varies along the standard lattice cell from its smallest value in the focusing in z-plane quadrupole QF to its largest value in the defocusing QD quadrupole. This is true because the horizontal beam size has a maximum value in QD and a minimum value in QF. The opposite situation occurs for the frequency of the vertical ion oscillation. Fig. 3 shows the instability zones (a) and the oscillation frequencies (b) for the heavy ions. We see that there will be locations with transversely stable ions along the ring for any atomic number $\boldsymbol{A}$ of the ion.

For the nominal current, the ratio of the total length of the stability zones to the ring circumference as a function of gap length for several ion atomic numbers was calculated from Eqs.(4.2), (3.8) and plotted in Fig. 4. In other words, Fig. 4. shows the percentage of ions with the transverse motion, stable in both $x, y$ planes in linear approximation, versus the gap length for average current $I=0.99 \mathrm{~A}$ in the HER. The design gap length in the High Energy Ring is chosen to be 88 bunch spacings or 5\% of the ring circumference. It corresponds to total length of the transverse stability zones on the order of $18 \%$ for typical atomic number $\boldsymbol{A}=\mathbf{2 0}$. A longer gap doesn't change this percentage drastically. 
The condition (4.2). is sensitive to small variations of parameters. The width of the stability zone is of the order

$$
\frac{\Delta \Omega}{\Omega} \geq \frac{1}{\left(\Omega T_{0}\right)^{2}(\Delta T / T)}
$$

or $\frac{\Delta \Omega}{\Omega} \simeq 10^{-3}$ while zones are separated by $\Omega T_{0}=\pi$. The locations of the stability zones would shift along the beam orbit as the current decays and/or the transverse beam sizes change.

The ion frequencies vary along the beam line mostly due to the variation of the rms beam sizes. The shift As along the accelerator produces the frequency variation

$$
\begin{aligned}
& \frac{\Delta \Omega_{x}}{\Omega_{x}}=-\frac{1}{2}\left(\frac{2 \sigma_{x}+\sigma_{y}}{\sigma_{x}\left(\sigma_{x}+\sigma_{y}\right)} \sigma_{x}^{\prime}+\frac{\sigma_{y}^{\prime}}{\sigma_{x}+\sigma_{y}}\right) \Delta s \\
& \frac{\Delta \Omega_{y}}{\Omega_{y}}==-\frac{1}{2}\left(\frac{2 \sigma_{y}+\sigma_{x}}{\sigma_{y}\left(\sigma_{x}+\sigma_{y}\right)} \sigma_{y}^{\prime}+\frac{\sigma_{x}^{\prime}}{\sigma_{x}+\sigma_{y}}\right) \Delta s
\end{aligned}
$$

where the sign ' means the derivative with respect to s. Comparison with (4.4) gives the width of the typical stability zone for the horizontal ion motion $w_{x}$ and vertical ion motion $w_{y}$ :

$$
\begin{aligned}
& w_{x}=-2 \sigma_{x} \frac{\Delta \Omega_{x}}{\Omega_{x}}\left[\frac{\sigma_{x}+\sigma_{y}}{\sigma_{x} \sigma_{y}^{\prime}+\left(262+\sigma_{y}\right) \sigma_{x}^{\prime}}\right] \mathbf{A} \cdot 0.6 \mathrm{~cm} \\
& w_{y}=-2 a \frac{\Delta \Omega_{y}}{\bar{\Omega}_{y}}\left[\frac{\sigma_{x}+\sigma_{y}}{\sigma_{y} \sigma_{x}^{\prime}+\left(2 \sigma_{y}+\sigma_{x}\right) \sigma_{y}^{\prime}}\right] \approx A \cdot 0.6 \mathrm{~cm}
\end{aligned}
$$

where the estimate uses the beam transverse rms sizes $\sigma_{x}=0.1 \mathrm{~cm}, \sigma_{y}=0.02 \mathrm{~cm}$ and the average Twiss parameters $\beta_{x}=1.5 .10^{3} \mathrm{~cm}$ and $\alpha_{x}=1.1$.

For transverse ion stability both the horizontal and vertical motion of the ion should be stable. The typical values of the transverse stability width $w$ for the standard straight cell of the HER are summarized in the third column of Table $\mathbf{3}$.

Another important parameter is the typical distance between the stability zones. According to Eq.(4.2) the shift of ion frequency on the order of

$$
\frac{\Delta \tilde{\Omega}_{x, y}}{\Omega_{x, y}}=\frac{\pi}{\Omega_{x, y}\left(T_{o}-\Delta T\right)}
$$

corresponds to the typical distance between the stability zones:

$$
\begin{gathered}
\Delta l_{x}=-2 \sigma_{x} \frac{\Delta \tilde{\Omega}_{x}}{\Omega_{x}}\left[\frac{\sigma_{x}+\sigma_{y}}{\sigma_{x} \sigma_{y}^{\prime}+\left(2 \sigma_{x}+\sigma_{y}\right) \sigma_{x}^{\prime}}\right] \approx \sqrt{A} \cdot 12.1 \mathrm{~cm} \\
\Delta l_{y}=-2 \sigma_{y} \frac{\Delta \tilde{\Omega}_{y}}{\Omega_{y}}\left[\frac{\sigma_{x}+\sigma_{y}}{\sigma_{y} \sigma_{x}^{\prime}+\left(2 \sigma_{y}+\sigma_{x}\right) \sigma_{y}^{\prime}}\right] \approx \sqrt{A} \cdot 27 \mathrm{~cm}
\end{gathered}
$$

for the HER standard lattice cell data. The distance between the stability zones is de- 
termined by $l_{x}$. The fourth column of Table $\mathbf{3}$ summarizes the typical distances between the stability zones for the transverse linear ion motion for the main ion species in the HER vacuum chamber.

According to Eq.(4.7),(4.8) the widest stability zone corresponds to the regions with small $\sigma_{x}^{\prime}, \sigma_{y}^{\prime}$, i.e. the quadrupole locations. We should use the parabolic term $\Delta s^{2}$ in the expansions $(4.5),(4.6)$ to find the width of the stability zone near the regions with $\sigma_{x}^{\prime}, \sigma_{y}^{\prime} \approx 0$. Therefore the width of the stability zone $\widetilde{w}$ near the quadrupole locations is proportional to $\sqrt{A}$. The fifth column of Table $\mathbf{3}$ shows the typical value of the stability zones $\widetilde{w}$ near the quadrupole locations.

Table 3. The characteristics of the transverse stability zones for the main ion species in the HER vacuum chamber. $w$ - the typical width of the stability zone

$\Delta l$ - the typical distance between the stability zones $\widetilde{w}$ the width of the stability zone near the QF,QD locations

\begin{tabular}{|c|c|c|c|c|}
\hline & A & $w, m$ & $\Delta l, \mathrm{~cm}$ & $\widetilde{w}, \mathrm{~cm}$ \\
\hline$H_{2}^{+}$ & 2 & 1.2 & 17 & 16 \\
\hline $\mathrm{CO}+$ & 28 & 17 & 64 & 56 \\
\hline $\mathrm{CO}_{2}^{+}$ & 44 & 27 & 80 & 75 \\
\hline $\mathrm{CH}_{4}^{+}$ & 16 & 9.9 & 48 & 44 \\
\hline
\end{tabular}

\subsection{Non-linear motion}

The ion motion is generally speaking not linear. The main effect of the nonlinear terms in the Hamiltonian is amplitude dependence of the ion frequencies $\Omega\left(A_{x}, A_{y}\right)$ and higher harmonics in the ion motion. At the amplitudes small compared to the rms beam sizes the frequencies of ion oscillations are:

$$
\frac{\Omega_{x}-\Omega_{x}(0)}{\Omega_{x}(0)}=-\frac{\sigma_{y}}{8\left(\sigma_{x}+\sigma_{y}\right)}\left[\frac{A_{y}^{2}}{\sigma_{y}^{2}}+\left(1+2 \frac{\sigma_{x}}{\sigma_{y}}\right) \frac{A_{x}^{2}}{2 \sigma_{x}^{2}}\right]
$$

where $\Omega(0)$ is frequency in the linear approximation. The frequency $\Omega_{y}$ can be obtained replacing indexes $x, y$. The phase advances for ions with the different amplitudes for the time of beam train are different. For example, for the ions with the amplitudes $0.1 \sigma_{x}$ and $0.5 \sigma_{x}$, the difference in the phase advances of horizontal oscillations is about $2.0 \mathrm{rad}$ for an ion with $\boldsymbol{A}=44$ and 9.4rad for an ion with $\boldsymbol{A}=\mathbf{2}$. Hence, if an ion with zero amplitude is stable, the amplitude of the order of $2 A^{2} /\left(\sigma_{x} \sigma_{y}\right) \simeq 210^{-3}$ is enough to cause the transverse instability of the ion.

The motion at small amplitudes can be modelled using Hamiltonian (3.10), describing long octupole, plus free motion within the gap. The corresponding map can be obtained by the second order canonical perturbation theory which is quite adequate for 
tracking with number of turns of the order of few hundred. Result of the simulation show that stability of ions with small but finite amplitudes corresponds to the criterion of the linear stability Eq. (4.2) with the amplitude dependent frequency Eq. (4.12), see Fig. 5. Higher harmonics of the ion motion do not change noticeable the stability of the motion.

Ions with non-zero amplitudes may have their own zones of stability, however, these zones, generally, are shifted longitudinally compared to zones of the linear theory. That reduces the number of ions stable both transversely and longitudinally.

The motion with large amplitudes is described by the Hamiltonian (3.11):

$$
H_{x}=\frac{p_{x}^{2}}{2 m_{i}}+\frac{e I Z_{o}}{4 \pi} \ln \left(\frac{x^{2}}{b_{o}^{2}}\right)
$$

with $b_{o}$ the typical radius of the beam pipe. The frequency of oscillations in the horizontal plane $\Omega_{x}\left(a_{x}\right)$, where $a$, is an ion amplitude, is

$$
\Omega_{x}\left(a_{x}\right) \simeq \Omega_{x}(0) \sqrt{\frac{\pi}{2} \frac{\sigma_{x}\left(\sigma_{x}+\sigma_{y}\right)}{a_{x}^{2}}}
$$

where $\Omega_{x}(0)$ is the frequency of the linear ion oscillation given by (3.8).

Typically, the motion with large amplitudes is unstable. Exception can be caused by trapping of ions within separatrices of the non-linear resonances. Small separatrices indeed has been observed (for different ring parameters) in simulations. For the PEP-XI, however, the resonance condition

$$
k \Omega_{x, y}=n \omega_{o},
$$

where $\omega_{0}=8.6 \cdot 10^{5} \frac{\mathrm{rad}}{\mathrm{sec}}$ is the revolution frequency in the HER and $k$ and $n$ are integers, means, for $\Omega_{x, y} \approx 3.3 \cdot 10^{7} \frac{\mathrm{rad}}{\mathrm{sec}}$ and $k=1$, that the order of the resonance is $n \approx 35$. The resonances of a such high order are strongly suppressed.

Effect of the gap with the length AT on the ion motion may be understood as the effect of the external periodic perturbation with frequencies at the harmonics of the revolution frequency within the band $\boldsymbol{A} \boldsymbol{w} \simeq \pm 1 /(\Delta T)$. If there are nonlinear resonances associated with these frequencies, they can be stable only if they do not overlapped. Otherwise, the motion become stochastic and ions are unstable. The variation of the amplitude AA changes the ion frequency by the frequency distance between resonances $1 / T_{\text {rev }}$ if $T_{\text {rev }}[\Omega(A+\mathbf{A A})-\Omega(A)] \simeq 1$. According to Eq. (4.12) the resonances overlap at the amplitudes

$$
A / \sigma \simeq 1 / \sqrt{\Omega T_{\text {rev }}}<<1
$$

Hence, the ion motion becomes stochastic at the amplitudes much smaller than rms beam size. 
The lifetime of unstable ions can be estimated assuming that the gap to gap phase advance of the ion oscillations $\mu=\mathrm{RT}$ are uncorrelated. The coordinates of an ion change during the passage of the gap from

$$
x_{0}=a_{0} \cos \mu_{0}, \quad x_{0}^{I}=-\Omega_{x} a_{0} \sin \mu_{0}
$$

to

$$
x=a_{0} \cos \mu_{0}-\Omega_{x} T_{g} a_{0} \sin \mu_{0}, \quad x^{\prime}=-\Omega_{x} a_{0} \sin \mu_{0} .
$$

The new amplitude is

$$
a^{2}=x^{2}+\left(x^{\prime} / \Omega_{x}\right)^{2}=a_{0}^{2}\left[1-\Omega_{x} T_{g} \sin \left(2 \mu_{0}\right)+\left(\Omega_{x} T_{g}\right)^{2} \sin ^{2} \mu_{0}\right] .
$$

For uncorrelated phases that gives the equation for the variation of the amplitude with the number of turns $n$ :

$$
\frac{d a^{2}}{d n}=\frac{1}{2}\left(\Omega_{x} T_{g}\right)^{2} a^{2} .
$$

For small amplitudes that gives exponential growth what could be expected for unstable motion. However, for large amplitudes the frequency (5.60) decreases with $a^{2}$ giving the linear growth

$$
\frac{d a^{2}}{d n}=\frac{\pi}{4}\left(\Omega_{x}(0) T_{g}\right)^{2} \sigma_{x}\left(\sigma_{x}+\sigma_{y}\right)
$$

Note, that ions drift faster horizontally.

A typical ion with $\boldsymbol{A}=\mathbf{4 4}, \sigma_{x}=1 \mathrm{~mm}$ can reach the horizontal aperture of the vacuum chamber $b_{0}=5 \mathrm{~cm}$ in $\boldsymbol{n}=\mathbf{2 5}$ turns. A Hydrogen ion reaches the wall of the vacuum chamber in one or two turns.

Therefore the typical lifetime of the ion in the vacuum chamber of the High Energy Ring is of order of several turns of the beam in the accelerator, i.e. near $30 \mu \mathrm{sec}$. This number is in good agreement with the computer simulation data. The ion tracking program simulated the transverse motion of an ion as a series of impulsive non-linear bunch-ion kicks with free drift in between kicks. It was found that the average lifetime of the ions, created inside the region $|x|<\sigma_{x},|y|<\sigma_{y}$ is of the order of $40 \mu \mathrm{sec}$.

\subsection{Distribution function of ions}

The adverse effects produced by the ions depend not only on the number of ions along the beam but on the transverse distribution of ions as well.

It was shown by Tavares ${ }^{[15]}$ that the transverse distribution of the ion cloud is narrower then the transverse distribution of the electron beam $\sigma_{e}$. If the residual gas temperature is small compared to the depth of the potential well, the equilibrium distribution is

$$
f(x)=\frac{1}{\pi \sqrt{2 \pi \sigma_{e}^{2}}} K_{0}(\xi) e^{-\xi}
$$

where $\xi=\left(x /\left(2 \sigma_{e}\right)\right)^{2}$. Such a narrow distribution substantially enhances the tune spread of betatron oscillations of electrons in the ring. 
It is worth noting, that amplitude distribution of stable ions is always the same as the averaged amplitude distribution of electrons.

\section{The longitudinal stability}

It was mentioned that a small variation of the ion oscillation frequency may turn the stable ion into an unstable one. The change of the ion frequency may be caused by the current decay, the longitudinal ion motion, or the space charge of the accumulated ions.

\section{1) The current decay}

The typical time of the current decay is given by the $\frac{1}{e}$ luminosity decay time ${ }^{[3]} \tau_{L}=$ $1.92 \mathrm{hr}$. The ion oscillation shift due to the current decay process may be written as

$$
\frac{\Delta \Omega_{y}}{\Omega_{y}}=\frac{1}{2} \frac{\Delta I(t)}{I}=-\frac{1}{2} \frac{\Delta t}{\tau_{L}}
$$

According to Eq. (4.4) the stable motion of the ion with the atomic number $\boldsymbol{A}=20$ will turn into an unstable one due to the current decay in a time of the order of

$$
\Delta t_{I}=\frac{47 \mathrm{~L}}{\Omega_{y} T_{o}} \operatorname{arccot}\left(\frac{\Omega_{y} \mathbf{A T}}{2}\right)=18 \mathrm{sec}
$$

with $T_{0}=7.3 \mu \mathrm{sec}$ the revolution period in the HER, AT $=0.37 \mu \mathrm{sec}$ the gap length, and $\Omega_{y}=4.1 \cdot 10^{7} \frac{\mathrm{rad}}{\mathrm{sec}}$ the frequency of the vertical oscillations for the ion with $\boldsymbol{A}=\mathbf{2 0}$.

2) The longitudinal motion of the ions

Generally speaking the ion is subjected to the longitudinal as well as the transverse motion in the field of the electron beam. There are several sources of the longitudinal ion motion in the accelerator : the variation of transverse beam sizes $\sigma_{x}, \sigma_{y}$ along the accelerator, the cross-field drift in the electromagnetic field of the beam, and the crossfield drift in the magnets due to electric field of the beam and magnetic fields of the quadrupole and bending magnets.

The equation of the longitudinal motion of a nonrelativistic ion in the electromagnetic field of the bunch is

$$
m_{\text {ion }} \ddot{s}=e E_{s}+\frac{e}{c}\left[\dot{y} H_{x}-\dot{x} H_{y}\right]
$$

First of all let us consider the first term on the right side of Eq.(5.3). The variation of the transverse beam sizes causes the modulation of the charge density of the beam along the ring and results in the longitudinal ion motion. The kick of the longitudinal velocity, $\Delta v_{s}$, of the single charged ion due to the electron bunch passage is given by

$$
\Delta v_{s}=\frac{e}{m_{i}} \int_{0}^{\sigma_{s} / c} E_{s}(x, s-c t, y, \tilde{s}) d t .
$$

with $m_{i}=\boldsymbol{A m}$, the ion mass, $\sigma_{s}$ the natural bunch length, and $E$, the longitudinal electric field of the bunch. 
Our main concern is the ions with small amplitude of transverse oscillations. The longitudinal electric field of the electron bunch near the closed orbit of the beam is given by Eq.(A.15)

$$
E,=-2 \rho_{o}(s-c t)\left(\frac{\sigma_{x}^{\prime}+\sigma_{\mathbf{z}}}{\sigma_{x}+\sigma_{y}}\right) .
$$

Therefore the kick of the longitudinal velocity of the ion with atomic number $A$ is

$$
\Delta v_{s}=-\frac{2 N_{B} r_{p} c}{A}\left(\frac{\sigma_{x}^{\prime}+\sigma_{y}^{\prime}}{\sigma_{x}+\sigma_{y}}\right)=-\frac{2 N_{B} r_{p} c}{A\left(\sigma_{x}+\sigma_{y}\right)}\left(\frac{\alpha_{x} \epsilon_{x}-\left(\eta \tilde{\sigma}_{\epsilon}\right)\left(\eta^{\prime} \tilde{\sigma}_{\epsilon}\right)}{\sigma_{x}}+\frac{\alpha_{y} \epsilon_{y}}{\sigma_{y}}\right)
$$

with $\alpha_{x, y}, \beta_{x, y}$ the Twiss parameters, $\epsilon_{x, y}$ the emittances, $\tilde{\sigma}_{\epsilon}$ the relative energy spread, $\eta, \eta^{\prime}$ the off-energy functions, $r_{p}$ the classical radius of the proton, and $N_{B}$ the bunch population. Note that the same expression may be found in a paper by D.Sagan ${ }^{[8]}$.

The typical value of the longitudinal ion kick due to the passage of the bunch for the High Energy Ring parameters is given by

$$
\Delta v_{s}=\frac{2 N_{B} r_{p} c}{A\left(\sigma_{x}+\sigma_{y}\right)} \frac{\alpha_{x} \epsilon_{x}}{\sigma_{x}}=\frac{115}{A} \frac{\mathrm{cm}}{\mathrm{sec}}
$$

with $\sigma_{x}+\sigma_{y}=0.12 \mathrm{~cm}, \alpha_{x}=1.13, \epsilon_{x}=48.24 \mathrm{~nm}-\mathrm{rad}$, and $\sigma_{x}=0.1 \mathrm{~cm}$. The direction of the longitudinal kick for the ion near the beam centerline is such that the kick pushes the ion in the direction of the local minimum of $\sigma_{\boldsymbol{x}}$, since the horizontal emittance is greater than the vertical one.

As we see, the typical value of the longitudinal kick is smaller than the kick of the transverse ion velocity given by Eq.(3.3). However the effect of the longitudinal kicks can accumulate from one bunch to another. Let us find the time $\Delta t_{l}$ it takes for an ion with atomic number $\boldsymbol{A}$ to leave the transverse stability zone due to the longitudinal motion. For the typical width $w$ of the transverse stability zone given by Table $\mathbf{3}$, the typical time $\Delta t_{l}$ may be written as

$$
\Delta t_{l}=\sqrt{\frac{w}{\Delta v_{s}} \frac{2 l_{b}}{c}}-A \cdot 6.6 \mu \mathrm{sec}=132 \mu \mathrm{sec}
$$

with $\Delta v_{s}$ given by Eq.(5.7), $l_{b}=126 \mathrm{~cm}$ the bunch spacing, and $w=12 \mathrm{~cm}$ the typical width of the stability zone for an ion with $\boldsymbol{A}=20$. We see that the typical time it takes for the ion to leave the transverse stability zone due to the longitudinal motion is of the order of ten revolution periods of the High Energy Ring.

The longitudinal ion motion may be described as the motion in the average potential of the beam. The depth of the potential well (3.2) varies along the accelerator, since the transverse beam sizes are functions of the longitudinal coordinate $s$. The variation of the beam potential along the standard straight cell of the HER is depicted on Fig 1. 
We can find the depth of the potential well for the longitudinal motion of the ions near the beam centerline“]:

$$
\Delta U=-\frac{A m_{p} c}{l_{b}} \int_{s_{1}}^{s_{2}} \Delta v_{s} d s=\frac{Z_{o} I}{2 \pi} \ln \left(\frac{\sigma_{x}\left(s_{2}\right)+\sigma_{y}\left(s_{2}\right)}{\sigma_{x}\left(s_{1}\right)+\sigma_{y}\left(s_{1}\right)}\right)
$$

where $Z_{0}=377 \Omega$ is the impedance of free space, $I$ is the current in the ring, and $s_{2}, s_{1}$ are the points of the maximum and minimum values of the expression $\sigma_{x}+\sigma_{y}$ respectively. For the HER parameters, $\Delta U=22 \mathrm{~V}$ (see Fig 1). The potential well has minimum at the defocusing quads in the straight sections where dispersion function is zero.

The largest velocity of the longitudinal ion motion due to the modulation of transverse beam cross-section along HER is given by

$$
v_{s}^{\max }=\sqrt{\frac{2 e \Delta U}{A m_{p}}}=\frac{1}{\sqrt{A}} 6.3 \cdot 10^{6} \frac{\mathrm{cm}}{\mathrm{sec}}
$$

According to Table 3, a shift of the longitudinal ion coordinate of several centimeters is sufficient to leave the transverse stability zone. The amplitude of unstable ions grows to large amplitudes, where the longitudinal velocity kick is less than we can expect from Eq.(5.6). The ion with non-zero transverse amplitude of oscillations feels a diminish average longitudinal acceleration. Therefore the ion can not reach such a large velocity as that given by Eq.(5.10).

Another source of the longitudinal ion motion is the longitudinal electric field caused by the variation of the beam pipe cross-section along the accelerator. The cross-section of the HER vacuum chamber is a round one with the inner radius $5 \mathrm{~cm}$ in the straight sections and octagonal, $9 \mathrm{~cm}$ wide by $5 \mathrm{~cm}$ high, in the $\operatorname{arcs}^{[3]}$. There are 12 transitions from an octagonal to round cross-section in the HER. The length of each transition is close to $25 \mathrm{~cm}$. It was calculated using the code POISSON that the potential well of the beam along the beam line is deeper in the arcs by $2.7 \mathrm{~V}$ than in the straight sections. Fig 6 shows the beam potential behavior between the last arc cell and the first straight cell of a sextant. We see that the variation of beam pipe cross-section doesn't result in new pockets of the trapped ions, but only distorts the beam potential.

Another source of the longitudinal motion of the ion is the thermal velocity. The one-dimensional thermal velocity of the ion is given by Eq.(3.4). For the ion with atomic number $\boldsymbol{A}=20$, the time it takes to leave the stability zone of width $\mathrm{w}=12 \mathrm{~cm}$ is

$$
\Delta t_{T}=\frac{w}{v_{T}}=w \sqrt{\frac{A m_{p}}{k T}}=330 \mu \mathrm{sec}
$$

Now let us consider the longitudinal ion motion generated by the magnetic field:

$$
\ddot{s}=\frac{e H_{x}}{m_{i} c} \dot{y}-\frac{e H_{y}}{m_{\text {ion }} c} \dot{x}
$$

Since the ion is non-relativistic $\left(\frac{\Delta v_{x}}{c} \sim 10^{-5}\right)$, we expect that its motion caused by the magnetic field of both the magnets or the beam, may be considered as a perturbation 
of the transverse ion motion given by

$$
\left\{\begin{array}{l}
x(t)=x_{0} \cos \left(\Omega_{x} t\right)+\frac{x_{0}}{\Omega_{x}} \sin \left(\Omega_{x} t\right) \\
y(t)=y_{0} \cos \left(\Omega_{y} t\right)+\frac{\dot{y}_{0}}{\Omega_{\varphi}} \sin \left(\Omega_{y} t\right)
\end{array}\right.
$$

with $x_{o}, y_{o}, \dot{x}_{o}, \dot{y}_{o}$ the initial conditions of the ion motion and $\Omega_{x, y}$ the frequencies of the linear ion oscillations given by Eq.(3.8). Let us show several applications of this perturbation theory.

1) The cross-field drift of the ion in the bending magnets: The magnetic field in the bending magnets of the HER is $B_{y}=0.1819 \mathrm{~T}$. Therefore after the integration of Eq.(5.12) we obtain

$$
\dot{s}(t)=\dot{s}_{o}-\omega_{b}\left(x(t)-x_{o}\right)
$$

with $\dot{s}_{o}$ the initial longitudinal velocity of the ion, $\omega_{b}=\frac{e B_{y}}{m_{i}}$ the cyclotron frequency in the bending magnetic field $B_{y}$, and $x(t)$ given by Eq.(5.13). After averaging over the period of the horizontal ion oscillation $\frac{2 \pi}{\Omega_{*}}$, we obtain the typical cross-field drift velocity of the ion in the bending magnets :

$$
v_{B}=\dot{s}_{o}+\omega_{b} x_{0}
$$

The exact solution is given by Y.Miyahara, K.Takayama and G.Horikoshi ${ }^{[9]}$ :

$$
v_{B}=\left(\omega_{b} x_{o}+\dot{s}_{o}\right) \frac{\Omega_{x}^{2}}{\omega_{b}^{2}+\Omega_{x}^{2}}
$$

Table 4 shows the cyclotron frequencies $\omega_{b}$, the horizontal ion oscillation frequencies $\Omega_{x}$, given by Eq.(3.8) and the cross-field velocities $v_{B}$ in the bending magnetic field for the

\begin{tabular}{|c|c|c|c|c|}
\hline & A & $\omega_{b}, \frac{1}{s e c}$ & $\Omega_{x}, \frac{1}{\sec }$ & $v_{B}, \frac{\mathrm{cm}}{\mathrm{sec}}$ \\
\hline$H_{2}^{+}$ & 2 & $8.7 \cdot 10^{6}$ & $4.7 \cdot 10^{7}$ & $8.7 .10^{5}$ \\
\hline $\mathrm{CO}+$ & 28 & $6.2 \cdot 10^{5}$ & $1.3 \cdot 10^{7}$ & $6.4 \cdot 10^{4}$ \\
\hline $\mathrm{CO}_{2}^{+}$ & 44 & $4.0 \cdot 10^{5}$ & $1.0 \cdot 10^{7}$ & $4.1 \cdot 10^{4}$ \\
\hline $\mathrm{CH}_{4}^{+}$ & 16 & $1.1 \cdot 10^{6}$ & $1.7 \cdot 10^{7}$ & $1.1 \cdot 10^{5}$ \\
\hline
\end{tabular}
main ion species in the HER vacuum chamber.

Table 4. The cross-drift parameters for the main ion species in the HER vacuum chamber. 
The result of the perturbation theory is in good agreement with the exact solution (5.16), since the following condition is satisfied : $\omega_{b} \ll \Omega_{x}$.

The time it takes for the ion with $\boldsymbol{A}=20$ to leave the transverse stability zone of width $\mathrm{w}=12 \mathrm{~cm}$ is of the order of

$$
\Delta t_{B}=\frac{w}{\sigma_{x} \omega_{b}}=\frac{w}{\sigma_{x}} \frac{A m_{p}}{e B_{y}}=A^{2} \cdot 0.345 \mu \mathrm{sec}=140 \mu \mathrm{sec}
$$

Generally speaking, an ion entering a bending magnet can be repelled by the dipole's fringe field ${ }^{[9]}$. The reflection condition is

$$
\dot{s}_{o}<\frac{\left|x_{o} \omega_{b}\right|}{\sqrt{2}}
$$

The width of the dipole's fringe field is of the order of the vacuum chamber aperture $a,=5 \mathrm{~cm}$. According to Table 3, the typical width of the transverse stability zone for the light ions is less than $a_{o}$. Therefore the process of the trapping of the light ions in the regions near the dipole's fringes, does not seem to be important for the HER. At the same time heavy ions may be trapped in the locations of the dipole's fringes.

2) The cross-field drift of the ions in the quadrupole magnets: To evaluate the drift velocity we consider the family of the HER quadrupole magnets with gradient $\boldsymbol{k}=0.08 \frac{\mathrm{f}}{\mathrm{cm}}$. Substitution of $B_{x}=k y, B_{y}=\boldsymbol{k z}$ into Eq.(5.12) and integration gives

$$
\dot{s}(t)=\dot{s}_{o}+\frac{e k}{2 m_{i}}\left(y^{2}(t)-y_{o}^{2}-x^{2}(t)+x_{o}^{2}\right)
$$

with $x(t), y(t)$ given by Eq.(5.33) and $\dot{s}_{o}$ the initial longitudinal velocity of the ion. Again, averaging over the period of the transverse ion oscillation gives the cross-field drift velocity of the ion in the quadrupole magnet :

$$
v_{q}=\dot{s}_{o}-\frac{e k}{4 m_{i}}\left(y_{o}^{2}-x_{o}^{2}-\frac{\dot{y}_{o}^{2}}{\Omega_{y}^{2}}+\frac{\dot{x}_{o}^{2}}{\Omega_{x}^{2}}\right) \approx \frac{1}{A} 1.85 \cdot 10^{4} \frac{\mathrm{cm}}{\mathrm{sec}}
$$

with $z=(x, y), z_{o}=\sigma_{z}$, and $\dot{z}_{o}, \dot{s}_{o}=0$. Note that the using of perturbation theory is

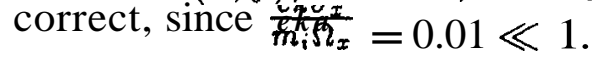

3) The cross-field drift in the electromagnetic field of the electron beam: According to Eq.(A.13), the magnetic field of the beam is given by

$$
H_{x}=E_{y}=-\frac{\partial U}{\partial y}, H_{y}=-E_{x}=\frac{\partial U}{\partial x}
$$

Since the ion motion is non-relativistic, we can apply perturbation theory. Substituting 
Table 5. The typical times for an ion to leave the transverse stability zone due to the main mechanisms of the longitudinal motion.

\begin{tabular}{lll}
\hline Mechanism & Symbol & time $\mu \mathrm{sec}$ \\
\hline Variation of $\sigma_{x}, \sigma_{y}$ & $\Delta t_{l}$ & $\mathbf{1 3 2}$ \\
Drift in the dipoles & $\Delta t_{B}$ & 140 \\
The thermal velocity & $\Delta t_{T}$ & 330 \\
Drift in the beam field & $\Delta t_{d}$ & $\mathbf{5 . 1} \cdot 10^{5}$ \\
\hline
\end{tabular}

(5.21) into Eq.(5.12) and noting that

$$
\frac{d U}{d t}=\frac{\partial U}{\partial x} \dot{x}+\frac{\partial U}{\partial y} \dot{y}
$$

after integration of Eq.(5.12) we obtain

$$
\dot{s}=\dot{s}_{o}-\frac{e}{m_{i} c}\left[U(x(t), y(t))-U\left(x_{0}, y_{0}\right)\right]
$$

The drift velocity may be obtained by averaging Eq.(5.23) over the typical time of the transverse ion oscillations.

For a small amplitude of ion oscillation the beam potential $U$ is given by Eq.(3.7)

$$
U(x(t), y(t))=\frac{m_{i}}{e}\left(\frac{\Omega_{x}^{2} x^{2}(t)}{2}+\frac{\Omega_{y}^{2} y^{2}(t)}{2}\right)
$$

Therefore the drift velocity in the electromagnetic field of the beam is

$$
v_{d}=\dot{s}_{o}-\frac{1}{4 c}\left(\dot{x}_{o}^{2}+\dot{y}_{o}^{2}\right)+\frac{1}{4 c}\left(\Omega_{x}^{2} x_{o}^{2}+\Omega_{y}^{2} y_{o}^{2}\right)=\frac{450}{A} \frac{\mathrm{cm}}{\mathrm{sec}}
$$

for the typical initial conditions of the ion $x_{0}=\sigma_{x}, y_{0}=\sigma_{y}$, and $\dot{x}_{0}, \dot{y}_{0}, \dot{s}_{0}=0$. The time it takes for the ion with $\boldsymbol{A}=\mathbf{2 0}$ to leave the stability zone of width $w=12 \mathrm{~cm}$ due to the cross-field drift in the field of the beam, is given by

$$
\Delta t_{d}=\frac{w}{v_{d}}=0.51 \mathrm{sec}
$$

Finally, Table 5 summarizes the typical times for the ion to leave the transverse stability zone due to the longitudinal motion. We see that the main mechanisms of longitudinal motion of the ions in the HER are the cross-field drift in the bending magnets, the longitudinal motion caused by the variation of the transverse beam sizes and the motion due the thermal velocity of the ion. 
Note that for the $s$ near the locations of quadrupole magnets, the processes of the longitudinal motic: are very weak. Moreover, according to Table $\mathbf{3}$, the width of the transverse stability zone near the quadrupole location, $\widetilde{w}$, is much greater than the typical width of the stability zone $\mathrm{w}$. We can expect that the ions will be trapped in such regions. However the accumulation of the ions will be stopped soon. The reason for this is the space charge of the ion cloud.

\section{3) The space charge of the accumulated ions}

The space charge of the cloud of the trapped ions will shift the ion frequency and turn the stable ion motion into an unstable one. Let us calculatt this effect for the HER parameters. The effect defines the number of accumulated sons at the minimum of the potential well. For simplicity, we assume here that the transverse distribution of the ion is a replica of the beam distribution, i.e. is given by

$$
\rho_{i}=\frac{\rho_{o i}}{2 \pi \sigma_{x} \sigma_{y}} \exp \left(-\frac{x^{2}}{2 \sigma_{x}^{2}}-\frac{y^{2}}{2 \sigma_{y}^{2}}\right)
$$

with $\rho_{o i}=\frac{N_{i w}}{w}$ the linear charge density of the ion cloud, $N_{i w}$ the number of ions accumulated in the stability zone with the width $\tilde{w}$, and $\sigma_{x}, \sigma_{y}$ the beam transverse rms sizes. According to Table 5 the typical width of the transverse stability zone near the location of the quadrupole magnets is of the order of $\widetilde{w}=50 \mathrm{~cm}$. This value is much greater than the beam transverse rms sizes $\sigma_{x}=0.1 \mathrm{~cm}, \sigma_{y}=0.02 \mathrm{~cm}$. Therefore we neglect the dependence of the electric field of the ion cloud on the longitudinal coordinate. Hence the equation for the potential of the in cloud is given by

$$
\nabla_{\perp}^{2} U_{i}=-4 \pi \rho_{i}
$$

with $\rho_{i}$ given by Eq.(5.27). The solution of this equation is given by (3.14) with $\rho_{\text {oi }}$ instead of $-e N_{e} / C$.

The linear charge density $\rho_{i o}$ of the accumulated ions, produces a shift of the ion frequency given by

$$
\frac{\Delta \Omega_{x, y}}{\Omega_{x, y}}=\frac{1}{2} \frac{\rho_{i}}{\rho_{o}}
$$

According to Eq.(4.2) the ion motion turns from a stable one into an unstable one at ion linear charge density

$$
\rho_{o i}=\frac{4 \rho_{o}}{\Omega_{x, y}\left(T_{o}-\Delta T\right)} \operatorname{arccot}\left(\frac{\mathbf{\&}, \mathbf{A} \mathbf{T}}{2}\right)
$$

For the accumulated ions with given atomic number $\boldsymbol{A}$, the time it takes to turn the ion motion into an unstable one ( the accumulation time ) is

$$
\Delta t_{a}=\frac{4 \tau_{\text {coll }}}{\Omega_{x, y}\left(T_{o}-\Delta T\right)} \operatorname{arccot}\left(\frac{\Omega_{x, y} \Delta T}{2}\right)
$$

with $\tau_{\text {coll }}$ the ionization time given by Table 1 . For the ions with atomic number $\boldsymbol{A}=20$ the ionization time is of the order of $\tau_{\text {coll }}=0.8 \mathrm{sec}$, and the frequency of the vertical 
oscillation is $\Omega_{y}=\mathbf{3 . 3} \cdot 10^{7} \frac{\mathrm{rad}}{\mathrm{sec}}$. Therefore from Eq.(5.31) we obtain

$$
\boldsymbol{A t}, \approx 2.3 m \mathrm{sec}
$$

Therefore, the time of ion accumulation in the High Energy Ring is of the order of one hundred revolution periods.

\section{Effects of the accumulated ions on the circulating beam.}

We estimate some of the consequences of the presence of ions on the electron beam in this Chapter.

The electric field of the ion cloud disturbs the betatron oscillation of an electron. The result is a spread and shift of the betatron frequency of the beam. All ions in the vacuum chamber of the High Energy Ring may be divided into three groups: one-turn ions, unstable ions, and trapped ions. Let us calculate the contribution of each group of ions to the tune shift and tune spread of the electron beam.

\subsection{One-turn ions.}

One-turn ions are the ions created during the last passage of the electron beam. These ions perform stable oscillations in the field of the electron beam because they "don't" know about the gap.

The number of one-turn ions is different for different bunches. Indeed, there are no one-turn ions for the first bunch of the beam, i.e. for the first bunch after the gap. At the same time the last bunch of the train of the electron bunches sees the ions generated by all previous bunches, namely

$$
N_{o t}=N_{e} \frac{T_{o}}{\tau_{\text {coll }}}=I \frac{T_{o}^{2}}{e \tau_{\text {coll }}}=1.3 \cdot 10^{9}
$$

with $T_{0}=7.3 \mu \mathrm{sec}$ the revolution period in the HER, $I=\frac{e N_{e}}{T}=0.99 \mathrm{~A}$ the total current in the ring, and $\tau_{\text {coll }}=0.25 \mathrm{sec}$ the total ionization time given by Eq.(2.2). Therefore, the ion cloud of one-turn ions will produce a bunch-to-bunch spread of the betatron tune.

According to Eq.(5.10) the frequency of the linear ion oscillation $\Omega_{x, y}$ is much greater than the revolution frequency $\omega_{0}=\frac{2 \pi}{T_{0}}$ and it is sufficient to consider the ion distribution averaged in time.

In the linear approximation the transverse distribution of the ions is the distribution given by Tavares ${ }^{[15]}$ at zero temperature because the ratio of the potential energy to the thermal energy of the ion

$$
\frac{U\left(\sigma_{x}\right)}{\frac{1}{2} k T}=\frac{A m_{p} \Omega_{x}^{2} \sigma_{x}^{2}}{k T}=\frac{e I Z_{o}}{2 \pi k T} \frac{\sigma_{x}}{\sigma_{x}+\sigma_{y}}
$$

is large, of the order of $1.9 \cdot 10^{\mathbf{3}}$ in $x$-plane and of the order $\mathbf{3 8 0}$ in the y-plane at $T=300 K^{\circ}$. We simulated the ion distribution generating 100 bunches equidistantly 
Table 6. The approximation parameters for

transverse distribution of the one-turn ions.

\begin{tabular}{|c|cccr|r|r|c|}
\hline$k$ & $\mid$ & $B_{k x}$ & & $\sigma_{k x} / \sigma_{x}$ & & $B_{k y}$ & $\sigma_{k y} / \sigma_{y}$ \\
\hline $\mathbf{1}$ & $\mid$ & $\mathbf{0 . 3}$ & $\mid$ & $\mathbf{0 . 0 3}$ & $\mathbf{0 . 3}$ & $\mathbf{0 . 0 6 2}$ \\
\hline $\mathbf{2}$ & $\mid$ & $\mathbf{0 . 3}$ & $\mid$ & $\mathbf{0 . 1 1 3}$ & $\mid$ & $\mathbf{0 . 3}$ & $\mathbf{0 . 1 8 5}$ \\
\hline $\mathbf{3}$ & $\mid$ & 0.4 & $\mid$ & 0.58 & & $\mathbf{0 . 4}$ & $\mathbf{0 . 6 7}$ \\
\hline
\end{tabular}

distributed along the ring with 500 particles per bunch. Initial distribution was Gaussian in $\mathrm{x}$ with the rms $\sigma=0.1 \mathrm{~cm}$ and $x^{\prime}=0$. The motion of each particle in the phase space corresponds to oscillations

$$
x(t)=x(0) \cos \left(\Omega_{J} t\right)+\frac{x^{\prime}(0)}{\Omega_{J}} \sin \left(\Omega_{J} t\right)
$$

with the amplitude dependent frequency $\Omega_{J}=\Omega(1-3 \epsilon J)$ where $\mathbf{J}=\Omega_{J} x(0)^{2}$ is defined by the initial conditions for the particle, $\mathbf{J}=\Omega x^{2}(0) /\left(1+3 \epsilon \Omega x^{2}(0)\right.$. The ion distribution Fig. 7 has a central core narrower than the beam conforming Tavares result Eq. (4.17). The nonlinearity $E$ does not affect the result even for $E$ big enough to produce filamentation during the time considered in the simulations. The transverse ion distribution Eq. (4.17) and the beam profile are depicted in Fig 8 for the total current in the ring $I=0.99 \mathrm{~A}$ and the beam size $\sigma_{x}=0.1 \mathrm{~cm}, \sigma_{y}=0.02 \mathrm{~cm}$.

To evaluate the betatron tune spread of the beam due to the one-turn ions we approximate the distribution given by Tavares by the sum of three Gaussian profiles :

$$
\rho_{o t}(x, y)=\frac{\operatorname{exc}^{\mathrm{n}} \mathrm{nC}}{\xi} \sum_{k=1}^{3} B_{k x} \exp \left(-\frac{x^{2}}{2 \sigma_{k x}^{2}}\right) \sum_{l=1}^{3} B_{l y} \exp \left(-\frac{y^{2}}{2 \sigma_{l y}^{2}}\right)
$$

with

$$
\xi \equiv \sum_{k=1}^{3} B_{k x} \sigma_{k x} \sum_{l=1}^{3} B_{l y} \sigma_{l y}
$$

Table 6 summarizes the coefficients of approximation $B_{k x}, B_{k y}, \sigma_{k x}$, and $\sigma_{k y}$. Note that $B_{k x}, B_{k y}$ are dimensionless parameters. The parameters $\sigma_{k x}$ and $\sigma_{k y}$ are given in units of the beam transverse rms sizes $\sigma_{x}$ and $\sigma_{y}$. It is easy to show that the charge distribution (6.6) satisfies the following condition

$$
\int_{-\infty}^{+\infty} \rho_{o t}(x, y) \cdot d x \cdot d y=e N_{o t}
$$


The electric field produced by the charge distribution (6.4) may be expanded for small $\frac{x}{\sigma_{x}^{i}}$ and $\frac{y}{\sigma_{y}^{i}}$. The linear term of expansion is

$$
E_{x}=\sum_{k l} \frac{2 e \rho_{k l}^{0} x}{\sigma_{k x}\left(\sigma_{k x}+\sigma_{l y}\right)}, \quad E_{y}=\sum_{k l} \frac{2 e \rho_{k l}^{0} y}{\sigma_{k y}\left(\sigma_{k x}+\sigma_{l y}\right)}
$$

where

$$
\rho_{k l}^{0}=\frac{N_{o t}}{C \xi} \sum_{k, l} B_{k x} \sigma_{k x} B_{l y} \sigma_{l y} .
$$

We may find the shift and spread of the betatron tune of the electron beam, $\Delta \nu_{k, l}$, produced by each term (6.7). The total value of the betatron tune spread is given by the sum over all $k$ and $l$ :

$$
\Delta \nu=\sum_{k=l}^{3} \sum_{l=1}^{3} \Delta \nu_{k, l}
$$

The betatron tune shift induced by a local quadrupole of strength $k(s)$ is given by

$$
\Delta \nu_{x, y}=\frac{1}{4 \pi} \oint_{C} \beta_{x, y}(s) k_{x, y}(s) d s
$$

where

$$
k_{x, y}(s)=\frac{e}{\gamma m_{e} c^{2}} \frac{\partial E_{x, y}}{\partial(x, y)}
$$

with $\gamma m_{e} c^{2}=9 \mathrm{GeV}$ the energy of electron in the High Energy Ring and $\beta_{x, y}$ the beta function. To evaluate the integral (6.16) we use the average values over the HER circumference of the parameters $\beta_{x, y}, \sigma_{x}^{i}, \sigma_{y}^{i}$. Therefore the betatron tune shift due to the ion cloud with a Gaussian profile is given by

$$
\Delta \nu_{y}=-\frac{N_{o t} r_{e}<\beta_{y}>}{2 \pi \gamma \xi} \sum B_{k x} B_{l y} \frac{\sigma_{k x} \sigma_{l y}}{\sigma_{l y}\left(\sigma_{k x}+\sigma_{l y}\right)}
$$

with $r_{e}=2.81 \cdot 10^{-13} \mathrm{~cm}$ the classical radius of the electron. The parameter $\xi=0.1 \sigma_{x} \sigma_{y}$ is the enhancement factor of the tune shift due to the ion distribution being narrower compared to the electron distribution. The average value of the $\beta$ function is $\beta_{x, y}=$ $1.5 \cdot 10^{3} \mathrm{~cm}$. Using the data of Table 6 we obtain the bunch-to-bunch spread of the betatron tune:

$$
\Delta \nu_{y}=0.02
$$

for $I=0.99 \mathrm{~A}$ the total current in the High Energy Ring. This estimate, based on the Tavaris distribution, is below the tune spread due to beam-beam interaction. 
According to the data of Table 6, Eq.(6.13) shows the shift of the betatron tune due to one-turn ions for an electron with small amplitudes of both the horizontal and vertical oscillation, namely, inside the region $|x|=0.03 \sigma_{x},|y|=0.062 \sigma_{y}$ in the plane of the transverse coordinates. In other words, only $0.02 \%$ of the total number of electrons in the HER have the betatron tune shift given by Eq.(6.13). It may be shown that for an electron with amplitudes of oscillations outside the region $|x|=0.58 \sigma_{x},|y|=0.67 \sigma_{y}$, the shifts of the betatron tunes are $\mathbf{A v},<8 \cdot 10^{-4}$ and $\Delta \nu_{y}<0.003$.

Now let us find the betatron tune spread caused by the first non-linear terms of the electric field of the ionic cloud of one-turn ions. First, we consider the contribution on the spread from each Gaussian term (6.4). A sum over all Gaussian profiles gives the total value of the spread of the betatron tune due to one-turn ions. The first nonlinear terms in the expansion of the field are

$$
\begin{aligned}
& \left.E_{x}^{k l}=-\frac{e N_{i}}{C} \frac{\rho f}{\left(\sigma_{x}^{i}+\sigma_{y}^{i}\right)^{2}} \underset{\sigma_{x}^{i}}{[}-\$+;(\mathrm{I}+\&) \$\right] \\
& E_{y}^{k l}=-\frac{e N_{i}}{C} \frac{\sigma_{x}^{i}}{\left(\sigma_{x}^{i}+\sigma_{y}^{i}\right)^{2}} \frac{y}{\sigma_{y}^{i}}\left[\frac{x^{2}}{\sigma_{x}^{i 2}}+\frac{1}{3}\left(1+2 \frac{\sigma_{y}^{i}}{\sigma_{x}^{i}}\right) \frac{y^{2}}{\sigma_{y}^{i 2}}\right] .
\end{aligned}
$$

The octupole component of the electric field couples the transverse betatron oscillation and produces the dependence of the betatron tune on the oscillation amplitude of the electron ( tune spread ). For the electron motion, written in the form

$$
\left\{\begin{array}{l}
x=a_{x} \sqrt{\beta_{x}(s)} \cos \left(\psi_{x}(s)+\psi_{x o}\right) \\
y=a_{y} \sqrt{\beta_{y}(s)} \cos \left(\psi_{y}(s)+\psi_{y o}\right)
\end{array}\right.
$$

where $\psi_{x}+\psi_{x o}, \psi_{y}+\psi_{y o}$ are the betatron phases and $a, a_{y},\left[\mathrm{~cm}^{\frac{2}{2}}\right]$ are the amplitudes of oscillations in terms of emittances, the value of tune spread may be written as ${ }^{(14)}$ :

$$
\begin{aligned}
& \delta \nu_{x}^{k l}=\frac{3 a_{x}^{2}}{32 \pi} \frac{e}{\gamma m_{e} c^{2}} \oint_{C} \beta_{x}^{2} \frac{\partial^{3} E_{x}^{k l}}{\partial x^{3}} d s+\frac{a_{y}^{2}}{16 \pi} \frac{e}{\gamma m_{e} c^{2}} \oint_{C} \beta_{x} \beta_{y} \frac{\partial^{3} E_{x}^{k l}}{\partial x \partial y^{2}} d s \\
& \delta \nu_{y}^{k l}=\frac{3 a_{y}^{2}}{32 \pi} \frac{e}{\gamma m_{e} c^{2}} \oint_{C} \beta_{y}^{2} \frac{\partial^{3} E_{y}^{k l}}{\partial y^{3}} d s+\frac{a_{x}^{2}}{16 \pi} \frac{e}{\gamma m_{e} c^{2}} \oint_{C} \beta_{x} \beta_{y} \frac{\partial^{3} E_{y}^{k l}}{\partial y \partial x^{2}} d s
\end{aligned}
$$

where

$$
\frac{\partial^{3} E_{x}^{k l}}{\partial x^{3}}=-\frac{e N_{i}}{C} \frac{2 \sigma_{y}^{i}}{\sigma_{x}^{i 3}\left(\sigma_{x}^{i}+\sigma_{y}^{i}\right)^{2}}\left(1+2 \frac{\sigma_{x}^{i}}{\sigma_{y}^{i}}\right)
$$




$$
\begin{aligned}
& \frac{\partial^{3} E_{y}^{k l}}{\partial y}--\frac{\mathrm{e} N_{i}}{C} \frac{2 \sigma_{x}^{i}}{\sigma_{y}^{i 3}\left(\sigma_{x}^{i}+\sigma_{y}^{i}\right)^{2}}\left(1+2 \frac{\sigma_{y}^{i}}{\sigma_{x}^{i}}\right) \\
& \frac{\partial^{3} E_{x}^{k l}}{\partial x \partial y^{2}}=\frac{\partial^{3} E_{y}^{k l}}{\partial y \partial x^{2}}=-\frac{e N_{i}}{C} \frac{2}{\sigma_{x}^{i} \sigma_{y}^{i}\left(\sigma_{x}^{i}+\sigma_{y}^{i}\right)^{2}}
\end{aligned}
$$

As earlier we use the average values of the beta function $\beta_{x, y}=1.5 \cdot 10^{3} \mathrm{~cm}$. Using the data of Table 6 gives the value of the spread of the betatron frequency, caused by the non-linearity of the electric field of the ion cloud of one-turn ions :

$$
\begin{gathered}
\delta \nu_{x}=\sum_{k=1}^{3} \sum_{l=1}^{3} \delta \nu_{x}^{k l}=0.002 \\
\delta \nu_{y}=\sum_{k=1}^{3} \sum_{l=1}^{3} \nu_{y}^{k l}=0.003
\end{gathered}
$$

These values are the shifts in the betatron tunes for an electron with amplitudes of oscillations given by $a_{x}=0.03 \sqrt{\epsilon_{x}}$ and $a_{y}=0.062 \sqrt{\epsilon_{y}}$, where $\epsilon_{x}=48.24 \cdot 10^{-7} \mathrm{~cm}-\mathrm{rad}$ and $\epsilon_{y}=1.93 \cdot 10^{-7} \mathrm{~cm}$-rad are the horizontal and vertical emittances respectively.

Finally, we see that the values of the bunch-to-bunch betatron tune spread $\boldsymbol{A} \boldsymbol{u}$, given by Eq.(6.13), and the tune spread due to non-linearity of the electric field of the cloud of one-turn ions $\delta \nu$ are less than the betatron tune spread caused by the beam-beam interaction. Moreover, only $0.2 \%$ of the total number of electrons in the HER will change its betatron tune by the value given by Eq.(6.20).

\subsection{Unstable ions.}

Unstable ions can not be removed from the orbit immediately. Therefore the electric field of the cloud of unstable ions may result in a shift of the betatron tune of the electron beam. First of all we note that the tune spread, caused by the non-linearity of the electric field of unstable ions, is negligible. Indeed it was shown in the previous chapter that the typical width of the cloud of unstable ions exceeds the value of the beam pipe radius after 3-4 turns of the beam in the HER. Hence the transverse distribution of such ions is uniform inside the vacuum chamber. The electric field of the uniformly distributed ion cloud is linear. Therefore it can not be the cause of the betatron tune spread of the electron beam $\delta \nu$.

To evaluate the betatron tune shift $\Delta \nu$ caused by unstable ions in the High Energy Ring we use the distribution which is the sum over the distribution functions of ions generated at the $n$-th turn. The $n$-th generation of ions is described by the Gaussian distribution with rms size

$$
a_{x, y}^{2}=\sigma_{x, y}^{2}+\Delta_{x, y}^{2} n
$$

where $\Delta_{x, y}^{2}=\Delta_{0}^{2} \sigma_{x, y}\left(\sigma_{x}+\sigma_{y}\right)$ and $\Delta_{0}^{2}=(\pi / 4)\left(\Omega_{0} T_{g}\right)^{2}$, Eq. (4.16). The tune shift given by this charge distribution is of the order of $10^{-4}$, by a factor $\left(\Omega_{0} T_{g}\right)^{2}$ smaller than the tune spread induced by the one-turn ions and much smaller than the beam-beam tune spread. 


\subsection{Trapped ions.}

A trapped ion is an ion with a stable transverse motion in the linear approximation. According to Chapter 4, the gap in the train of the electron bunches makes $82 \%$ of the total number of $\mathbf{A}=\mathbf{4 4}$ ions in the HER linearly unstable. At the same time $18 \%$ of these ions stay stable. Stable ions may accumulate from one turn to another.

All ions with a stable transverse motion may be divided into two groups: ions with a stable longitudinal motion and ions with an unstable longitudinal motion. Let us consider the shift and spread of the betatron tune induced by each group of trapped ions.

a) Trapped ions with a stable longitudinal motion.

The trapped ions with a stable longitudinal motion may accumulate near the location of the defocusing in x-plane quadrupole magnets QD, corresponding to the bottom of the beam potential well ( see Fig 1), and near the location of the dipole's fringes. We will consider the worst case when stability zones for a heavy ion with atomic number $\mathbf{A}=44$ coincide with the bottom of the beam potential well. According to Table $\mathbf{3}$, the typical width of the stability zone near the quadrupole locations is $\widetilde{w}=72 \mathrm{~cm}$ for an ion with atomic number $\mathbf{A}=\mathbf{4 4}$. At the same time the typical width of the fringe field of the dipoles is of the order of the vacuum chamber aperture, i.e. $5 \mathrm{~cm}$. Therefore we can neglect the process of accumulation of ions near the locations of the dipole's fringes.

There are two mechanisms which restrict the number of accumulated ions at the locations of quadrupole magnets: the non-linearity of the field of the electron beam and the space charge of accumulated ions. It is worth noting that secondary ionization of stable ions is equivalent to reduction of their atomic number by a factor of $\mathbf{2}$ and may make them unstable. However, this process is very slow and can be neglected.

Let us assume that an ion with zero amplitude of oscillations is stable. The nonlinearity of the field of the electron beam results in the dependence of the ion's oscillation frequency on the amplitudes of the oscillations given by Eq.(4.12). By using Eq.(4.4), we may find the dimensions of the area in which an ion may be trapped :

$$
\begin{aligned}
& a_{1}=\sigma_{x} \sqrt{\frac{8 \sigma_{y}}{\left(\Omega_{2} T_{o}\right)^{2}\left(A T / T_{0}\right) \sigma_{x}}} \\
& a_{y}=4 \sigma_{y} \sqrt{\frac{16}{\left(\Omega_{y} T_{0}\right)^{2}\left(\Delta T / T_{0}\right)}}
\end{aligned}
$$

These dimensions may be considered as the typical widths of the cloud of trapped ions.

The tune shift produced by the ions is maximum for electrons with small amplitudes. The electric field Eq. (6.7) generated by the trapped ions at small $x, y$

$$
E_{x}=2 e n_{i} y, \quad E_{x}=2 e n_{i}\left(\frac{\sigma_{y}}{{ }_{*}}\right) x
$$

depends on the ion density $n_{i}$ rather then the total number of trapped ions. If ions were accumulated at small amplitudes until the beam at these distances become neutral, that 
is up to $n_{i}=n_{e}$, the tune shift for electrons with small amplitudes would be several units and such electrons were lost in few revolution. The fraction of such electrons is small, of the order of

$$
(\sigma / a)_{x, y}^{2} \simeq\left(\frac{2.10^{-2}}{\sqrt{A}}\right)^{2}
$$

However, the phase space of the small amplitudes would be filled up continuously due to synchrotron radiation, intrabeam scattering, or interaction with residual gas until all electrons were lost. Although the process may be slow the situation is undesirable.

Fortunately, the space charge of accumulated ions makes ions unstable much earlier than neutralization takes place. Accumulated ions change effective beam density. The ion frequencies (3.8) vary with the beam density as

$$
\frac{\Delta \Omega_{x}}{\Omega_{x}} \simeq \frac{1}{2} \frac{\Delta n_{e}}{n_{e}} \frac{\sigma_{y}}{\sigma_{y}}, \quad \Delta \Omega_{y} \simeq \frac{1}{2} \frac{\Delta n_{e}}{n_{e}}
$$

Maximum variation of the $\Delta \Omega / \Omega$ for a stable ions is given by Eq. (4.4). Hence, the ions become unstable when the ion density is

$$
n_{i}=n_{e}\left(\frac{\triangleq n_{e}}{n_{e}}\right), \quad \text { where } \quad \frac{\Delta n_{e}}{n_{e}}=\frac{2 \sigma_{y}}{\sigma_{x}} \frac{1}{\left(\Omega_{x} T_{o}\right)^{2}\left(\Delta T / T_{o}\right)}
$$

The electron tune shift (6.10) given by these ions is

$$
\Delta \nu_{y}=\frac{r_{e} n_{i} C}{2 \pi \gamma}\left(\frac{l}{C}\right) \beta_{y}
$$

where the total number of trapped ions is

$$
n_{i} C=\frac{N_{e}}{\sigma_{x} \sigma_{y}} \frac{\Delta n_{e}}{n_{e}}
$$

and $\beta_{y}$ has to be taken at the location of the stable zones. The stable zones in the worst case have the total length $l=n_{Q} \widetilde{w}$, where $\widetilde{w}=72 \mathrm{~cm}$ is the width of the stability zone near the locations of horizontally defocusing quadrupoles and $n_{Q}$ is their number. Taking the ring circumference $C=2.2 \cdot 10^{5} \mathrm{~cm}$, and the number of lattice cells in the ring $n_{Q}=144$ we get $l / C=4.710^{-2}$. This number is, certainly, overestimated because quads in the ring (in dispersion suppressors, arcs, straight sections) are not identical.

That gives

$$
\Delta \nu_{y}=\frac{N_{e} r_{e} \beta_{y}}{2 \pi \gamma \sigma_{x} \sigma_{y}}\left(\frac{l}{C}\right) \frac{\Delta n_{e}}{n_{e}} .
$$

Using the total number of electrons in the ring $N_{e}=4.5 \cdot 10^{13}, \beta_{y}=25 \mathrm{~m}$, and the rms beam's transverse dimensions at the locations of QD magnets in straight sections $\sigma_{x}=0.07 \mathrm{~cm}$ and $\sigma_{y}=0.022 \mathrm{~cm}$ we obtain for an ion with atomic number $\boldsymbol{A}=\mathbf{4 4}$

$$
\Delta \nu_{y}=0.015
$$

All ions together may double this number. 
Now let us find the betatron tune spread caused by the non-linearity of the electric field of the cloud of trapped ions. For an ion with amplitudes of oscillations $a$, $=\sigma_{x}^{i}$ and $a_{y}=\sigma_{y}^{\imath}$, the change of the betatron tune is given by

$$
\begin{gathered}
\delta \nu_{x}^{t r}=\frac{3 \sigma_{x}^{i 2}}{32 \pi} \frac{e \widetilde{w} n}{\gamma m_{e} c^{2}} \widetilde{\beta}_{x} \frac{\partial^{3} E_{x}}{\partial x^{3}}+\frac{\sigma_{y}^{i 2}}{16 \pi} \frac{e \widetilde{w} n}{\gamma m_{e} c^{2}} \widetilde{\beta}_{x} \frac{\partial^{3} E_{x}}{\partial x \partial y^{2}}=0.0017 \\
\delta \nu_{y}^{t r}=\frac{3 \sigma_{y}^{i 2}}{32 \pi} \frac{e \widetilde{w} n}{\gamma m_{e} c^{2}} \widetilde{\beta}_{y} \frac{\partial^{3} E_{y}}{\partial y^{3}}+\frac{\sigma_{x}^{i 2}}{16 \pi} \frac{e \widetilde{w} n}{\gamma m_{e} c^{2}} \widetilde{\beta}_{y} \frac{\partial^{3} E_{y}}{\partial y \partial x^{2}}=0.02
\end{gathered}
$$

with $n=144$ the number of lattice cells in the HER, $\sigma_{x}^{i}$ and $\sigma_{y}^{i}$ the widths of the cloud of trapped ions given by Eq.(6.27), and E, E, the octupole component of the electric field of the cloud of trapped ions given by

$$
\begin{gathered}
\frac{\partial^{3} E,}{\partial x^{3}}=-\frac{e N_{t r}}{\widetilde{w} n} \frac{2 \sigma_{y}^{i}}{\sigma_{x}^{i 3}\left(\sigma_{x}^{i}+\sigma_{y}^{i}\right)^{2}}\left(1+2 \frac{\sigma_{x}^{i}}{\sigma_{y}^{i}}\right)=150 \frac{k V}{c m^{4}} \\
\frac{\partial^{3} E_{y}}{\partial y^{3}}=-\frac{e N_{t r}}{\widetilde{w} n} \frac{2 \sigma_{x}^{i}}{\sigma_{y}^{i 3}\left(\sigma_{x}^{i}+\sigma_{y}^{i}\right)^{2}}\left(1+2 \frac{\sigma_{y}^{i}}{\sigma_{x}^{i}}\right)=10200 \frac{k V}{c m^{4}} \\
\frac{\partial^{3} E_{x}}{\partial x \partial y^{2}}=\frac{\partial^{3} E_{z}}{\partial y \partial x^{2}}=-\frac{e N_{t r}}{\widetilde{w} n} \frac{2}{\sigma_{x}^{i} \sigma_{y}^{i}\left(\sigma_{x}^{i}+\sigma_{y}^{i}\right)^{2}}=320 \frac{k V}{\mathrm{~cm}^{4}}
\end{gathered}
$$

Finally we see that the shift and spread of the betatron tune of the electron beam induced by trapped ions with a stable longitudinal motion, are of the order of the value obtained from the beam-beam interaction. At the same time, the cloud of trapped ions does not change the betatron tune of an electron with amplitudes of oscillations outside the region $|\mathbf{x}|=0.27 \sigma_{x}$ and $|y|=0.180$, In other words, only $5 \%$ of the total number of electrons will have the betatron tune shift given by Eqs.(6.32) and (6.33). Since the ions will be trapped at the locations of the defocusing in $\mathrm{x}$-plane quadrupoles, utilization of the clearing electrodes may be useful.

\section{b) Trapped ions with an unstable longitudinal motion.}

The last group of ions is the transversely stable but longitudinally unstable ions. Such ions can not leave the stability zone immediately. Therefore, trapped ions with an unstable longitudinal motion, may result in a shift and spread of the betatron tune of the beam.

Again, the non-linearity of the electric field of the electron beam results in the dependence of the ion's oscillations frequencies on the amplitudes of oscillations. By 
using Eq.(6.25), we obtain the typical transverse sizes of the cloud of trapped ions:

$$
\begin{aligned}
& a,=4 \sigma_{x} \sqrt{\frac{4}{\Omega_{x}^{2} \Delta T\left(T_{o}-\mathbf{A T}\right) 2 \sigma_{x}+\sigma_{y}}}=\sqrt{A} \cdot 0.0550,=\sqrt{A} \cdot 0.0055 \mathrm{~cm} \\
& a_{y}=4 \sigma_{y} \sqrt{\frac{4}{\Omega_{y}^{2} \Delta T\left(T_{o}-\mathbf{A T}\right) 2 \sigma_{y}+\sigma_{x}}}=\sqrt{A} \cdot 0.031 \sigma_{y}=\sqrt{A} \cdot 0.0006 \mathrm{~cm}
\end{aligned}
$$

with $T_{o}=7.3 \mu \mathrm{sec}$ the revolution period in the HER, $\mathbf{A T}=0.37 \mu \mathrm{sec}$ the gap length, $\sigma_{x}=0.1 \mathrm{~cm}, \sigma_{y}=0.02 \mathrm{~cm}$ and $\Omega_{x}$ and $\Omega_{y}$ given by Eq.(3.8).

First of all let us find the equilibrium number of trapped ions with an unstable longitudinal motion inside one stability zone. The typical width of the stability zone w is given by Eq.(4.7). The time it takes for an ion with atomic number $\boldsymbol{A}$ to pass through the stability zone $\tau_{l}$ is given by Eq.(5.8). These characteristics are summarized in Table 7. The number of ions $N_{w}$, inside the stability zone with length $w$ and transverse widths $\sigma_{x}^{i}$ and $\sigma_{y}^{i}$, satisfies the following equation:

$$
\frac{d N_{w}^{i}}{d t}=\frac{N_{e}}{\tau_{\text {coll }}} \frac{w a_{x} a_{y}}{C \sigma_{x} \sigma_{y}}-\frac{N_{w}^{i}(t)}{\tau_{l}}
$$

with $N_{e}=4.5 \cdot 10^{13}$ the total number of electrons in the ring and $\tau_{\text {coll }}$ the ionization time for an ion species with atomic number $\boldsymbol{A}$ as given in Table 1. The first term of Eq.(6.41) describes the creation of ions by the electron beam. The second term describes the ions which leave the stability zone due to their longitudinal motion. The equilibrium number of ions inside the stability zone may be obtained from the following condition:

$$
\frac{d N_{w}^{t}}{d t}=0
$$

The result is

$$
N_{w}^{i}=N_{e} \frac{\tau_{l}}{\tau_{\text {coll }}} \frac{w \sigma_{x}^{i} \sigma_{y}^{i}}{C \sigma_{x} \sigma_{y}}
$$

The fifth column of Table 7 shows the equilibrium number of ions inside the stability zone.

Now we can find the total number of trapped ions with an unstable longitudinal motion in the ring. According to Fig 4, the total length of the stability zones $l_{s t}$ varies from $6 \%$ of the ring circumference for an ion with atomic number $\boldsymbol{A}=2$ to $22 \%$ of the ring circumference for an ion with $\boldsymbol{A}=44$. Therefore the total number of trapped ions with an unstable longitudinal motion is given by

$$
N_{i}=N_{w} \frac{l_{s t}}{w}=N_{e} \frac{\tau_{l}}{\tau_{\text {coll }}} \frac{l_{s t} a_{x} a_{y}}{C \sigma_{x} \sigma_{y}}
$$

with $\boldsymbol{a}$, and $a_{y}$ given by Eqs.(6.39) and (6.40). The third column of Table 11 shows this parameter for the main ionic species in the accelerator. Finally, we can find the shift of 
Table 7. The main characteristics

of the ions in the stability zone.

$w$ - the width of the stability zone

$\tau_{l}-$ the time it takes to pass through the zone

$N_{w}$ - the equilibrium number of ions in the zone

\begin{tabular}{|c|cccccr|c|}
\hline & & $\mathrm{A}$ & $\mid$ & $w, c m$ & & $\tau_{l}, \mu \mathrm{sec}$ & $N_{w}$ \\
\hline$H_{2}^{+}$ & $\mid$ & 2 & $\mid$ & $\mathbf{1 . 2}$ & $\mid$ & $\mathbf{1 3 . 2}$ & 14 \\
\hline$C O+$ & $\mid$ & 28 & $\mid$ & 17 & $\mid$ & 185 & $\mathbf{3 . 9 . 1 0 ^ { 4 }}$ \\
\hline$C O_{2}^{+}$ & $\mid$ & 44 & $\mid$ & 27 & $\mid$ & 290 & $\mathbf{1 . 2 . 1 0 ^ { 5 }}$ \\
\hline$C H_{4}^{+}$ & 16 & $\mid$ & 9.9 & $\mid$ & 106 & $2.2 \cdot 10^{3}$ \\
\hline
\end{tabular}

the betatron tune of the beam by using Eqs.(6.14) and (6.16). Table 8 shows the values of the shift of the betatron tune $\Delta \nu_{x}$ and $\Delta \nu_{y}$ induced by trapped ions with an unstable longitudinal motion.

Table 8 . The shift of the betatron tune.

induced by trapped ions with an unstable longitudinal motion.

$N_{i}$ - the total number of trapped ions

with unstable longitudinal motion

\begin{tabular}{|l|c|r|r|r|r|}
\hline & $\mathrm{A}$ & $N_{i}$ & $\Delta \nu_{x}$ & $\Delta \nu_{y}$ \\
\hline$H_{2}^{+}$ & 2 & $1.54 \cdot 10^{5}$ & $8.5 \cdot 10^{-6}$ & $7.8 \cdot 10^{-5}$ \\
\hline$C O^{+}$ & 28 & $9.5 \cdot 10^{7}$ & $3.8 \cdot 10^{-4}$ & 0.0035 \\
\hline$C O_{2}^{+}$ & & 44 & $2.12 \cdot 10^{8}$ & $5.35 \cdot 10^{-4}$ & 0.005 \\
\hline$C H_{4}^{+}$ & 16 & $7.23 \cdot 10^{6}$ & $5 \cdot 10^{-5}$ & $4.6 \cdot 10^{-4}$ \\
\hline
\end{tabular}

Therefore the shift of the betatron tune induced by trapped ions with an unstable longitudinal motion is given by

$$
\left\{\begin{array}{l}
\Delta \nu_{x}=9.7 \cdot 10^{-4} \\
\Delta \nu_{y}=0.009
\end{array}\right.
$$

We also can calculate the spread of the betatron tune of the beam by using Eqs.(6.21) and (6.22). The result is

$$
\left\{\begin{array}{l}
\delta \nu_{x}=6.5 \cdot 10^{-4} \\
\delta \nu_{y}=0.006
\end{array}\right.
$$

We see that the shift and spread of the betatron tune induced by trapped ions with an unstable longitudinal motion are much less than the shift and spread generated by ions trapped near the bottom of the beam potential well. 


\section{Conclusion and some comments}

The main contribution to the shift and spread of the betatron tune of the beam is caused by trapped ions with a stable longitudinal motion. The (conservative) value of the tune spread, $\boldsymbol{A u},=0.03$ is of the order of the tune shift due to the beam-beam interaction. However as compared to the shift due to the beam-beam interaction, only $5 \%$ of the total number of the electrons in the ring will change the betatron tune by the value $\Delta \nu_{y}=0.03$. The value of the spread and shift of the betatron tune of the beam induced by one-turn ions is comparable to the corresponding values of the shift and spread due to trapped ions.

The shift due to one-turn ions is unavoidable, since it is impossible to have the transverse clearing field everywhere around the circumference of the ring. Note that the betatron tune will be shifted by the value 0.02 only for $0.25 \%$ of the total number of the electrons in the High Energy Ring. In reality this number may be less if we take into account the bunched structure of the beam.

The effects concerning trapped ions with a stable longitudinal motion may be reduced by using the clearing electrodes at the locations of the defocusing in $\mathrm{x}$-plane quadrupole magnets. It may be proposed to use the vertical DC electric field since the vertical aperture of the vacuum chamber in the arcs is less than the horizontal one. The value of the clearing field must be greater than the trapping field, that is

$$
E_{c}>\frac{Z_{o} I}{\pi\left(\sigma_{x}+\sigma_{y}\right)}
$$

with $Z_{0}=377 \Omega$ the impedance of free space and $I$ the average beam current. For an average current of $I=0.99 \mathrm{~A}$, this would require a clearing field in excess of $1.0 \frac{\mathrm{kV}}{\mathrm{cm}}$.

Clearing electrodes usually undesirable adding cost and increasing the total coupling impedance. However, they can be made as passive matched lines used sometimes for damping of the transverse instabilities ${ }^{[16]}$. To avoid cross talk between bunches, the matched lines should be shorter than a quarter of the bunch spacing.

Comparing the effects of one-turn ions and that of the trapped ions it is worth noting that they scale differently with the revolution period $T_{0}$ of the machine. For a given current, the effect of one turn ions growth as $T_{0}^{2}$ while the width of a zone of stability shrinks as $\left(1 / T_{0}\right)^{2}$. The number of zones increases with $T_{0}$ but it doesn't matter because only zones at the minimum of the potential well are relevant. Hence,for large rings one-turn ions may dominate. In such a case, clearing electrodes can not help. The situation can be controlled using not one but $\mathbf{2}$ asymmetrically placed gaps, although $\mathrm{RF}$ transients in this case may require special study. It should be noted also that the bunch to bunch tune spread generated by one-turn ions may have positive effect on the coupled bunch instabilities and not necesseraly undesirable.

Several questions which are outside the scope of this paper concern the interactions between the beam and the cloud of ions. As long as the ion trace of the bunch carries information about its coherent oscillations, it can cause at least a multi-bunch instability of the beam ( see, for instance, reference""'). As an example, let us consider the longitudinal coupled bunch instability. Following the reference ${ }^{[1]} l e t n$ be the coupled bunch mode number. The coupled bunch dipole mode has spectral lines at the following 
frequencies:

$$
f_{p, n}=(n+p M) f_{o}+f_{s}
$$

with $-\infty<p<\infty$, the number of populated funches in the HER $M=1658$, the revolution frequency $f_{0}=140 \mathrm{kHz}$, and the synchrotron frequency of the accelerator $f_{s}=7.1 \mathrm{kHz}$. Note that $f_{p, n}$ runs over both positive and negative frequencies.

The frequencies of the linear ion oscillations may be obtained from Eqs.(4.4) and (4.5):

$$
f_{x}=\frac{\Omega_{x}}{2 \pi}=\frac{1}{\sqrt{A}} 11 M H z, \quad f_{y}=\frac{\Omega_{y}}{2 \pi}=\frac{1}{\sqrt{A}} 24 M H z
$$

The linear oscillation of an ion can excite the mode with $p=0$ and $n \sim 100$. According to Pedersen, the growth rate for the mode with such a large $n$ is less than the radiation damping time ( see Table 1 in reference'"']). Therefore the linear ion oscillations can not result in the longitudinal coupled beam instabilities.

It is worth noting also that ions will not be trapped at the location of the interaction point of the High Energy Ring. This is true since the current of the positron beam is twice as much as the current of the electron beam, while the transverse sizes of both the electron and positron beams are equal at the IP. Therefore the transverse motion of an ion near the location of the IP will be an unstable one.

To avoid the problems during the injection process it may be recommended to fill the bucket with the design number of particles per bunch $N_{B}=2.75 \cdot 10^{10}$ before going to the next bucket. Moreover, this injection scenario allows us to avoid the appearance of strong non-linear resonances. The reason for this is that the ion's oscillation frequencies will have the design values and, therefore, the non-linear resonances (3.13) will be strongly suppressed.

One more recommendation concerning the injection scenario may be given. The best way to fill the ring is the sequential filling, i.e. the filling from one bucket to the next sequentially. This will correspond to a large value of the gap length AT ( see Fig.4). At the same time, there are also no problems with the present design injection scenario ${ }^{[3]}$.

Finally, we want to mention that unstable ions in HER and electrons in the positron ring eventually hit the wall of the beam pipe and may produce secondary particles and heating of the vacuum pipe. The effect however, is small. For example, the power deposited by $210^{14} 200 \mathrm{eV}$ ions produced per second in HER is only about $5 \mathrm{~mW}$.

\section{Acknowledgements.}

The main part of this paper is a diploma written by one of us (D. V.) during his visit of SLAC. D. V. has the pleasure to thank Jonathan Dorfan and Mike Zisman for having suggested the problem considered here and to SLAC and its PEP-11 R\&D group for their hospitality. We are grateful to Martin Donald, Phil Morton, Charles Perkins, Richard Early, and J Langton for discussions and help with the B-factory data, and Tom Knight for a careful reading of the manuscript and his general computer assistance.

\section{Appendix A. The electromagnetic field of an electron bunch.}

Let us calculate the electromagnetic field of an electron bunch. 
The charge density $\boldsymbol{p}$ and the current density $\vec{j}=\vec{v} \rho$ of a bunch are the functions of the transverse coordinates and the parameter $\mathrm{s}-v t$, where $s$ is the position of the bunch center in the ring:

$$
\rho=\rho(x, s-v t, y, \widetilde{s}) .
$$

The variable $\tilde{s}$ describes the slow variation of the charge distribution $p$ along the ring caused by the variation of the transverse rms beam sizes $\sigma_{x}$ and $\sigma_{y}$ along the machine and can be considered as adiabatic parameter.

The Maxwell's equation for the electric field $\vec{E}$ is

$$
\nabla^{2} \vec{E}-\frac{1}{c^{2}} \frac{\partial^{2} \vec{E}}{\partial t^{2}}=4 \pi \nabla \rho+\frac{4 \pi}{c^{2}} \frac{\partial \vec{j}}{\partial t}
$$

The frequency harmonics of $\vec{E}$

$$
\vec{E}_{\omega}(x, s, y, \widetilde{s})=\frac{1}{2 \pi} \int_{-\infty}^{\infty} \vec{E}(x, s-v t, y, \widetilde{s}) e^{i \omega t} d t=e^{i \omega \frac{s}{v}} \frac{\vec{E}_{\omega}(x, y, \widetilde{s})}{v}
$$

satisfy, in the ultra-relativistic case, the equation

$$
\begin{gathered}
\nabla_{\perp}^{2} \vec{E}_{\omega}^{\perp}(x, y, \widetilde{s})=4 \pi \nabla_{\perp} \rho_{\omega}(x, y, \widetilde{s}) \\
\nabla_{\perp}^{2} E_{\omega}^{s}(x, y, \widetilde{s})=4 \pi \frac{\partial \rho_{\omega}(x, y, \widetilde{s})}{\partial \widetilde{s}}
\end{gathered}
$$

It is easy now to go back to the time domain, where the field is given by the potential $U, \vec{E}=-\mathrm{VU}$, which satisfies the equation

$$
\nabla_{\perp}^{2} U=-4 \pi \rho(x, s-\mathrm{vt}, y, \widetilde{s})
$$

The solution of the eq.(A.6) with the boundary condition $U=0$ on the wall of the vacuum chamber, may be written in the form of

$$
U=U_{1}+U_{2},
$$

with $U_{1}$ given by the inhomogeneous equation in the free space

$$
\nabla_{\perp}^{2} U_{1}=-4 \pi \rho
$$


and $U_{2}$ satisfying the Laplas equation

$$
\nabla_{\perp}^{2} U_{2}=0
$$

with the boundary condition

$$
\left.U_{2}\right|_{\text {wall }}=-\left.U_{1}\right|_{\text {wall }}
$$

For a Gaussian transverse profile of the bunch, the charge density $\rho$ is given by

$$
\rho(x, s-v t, y, \tilde{s})=-\frac{\rho_{o}(s-v t)}{2 \pi \sigma_{x} \sigma_{y}} \exp \left(-\frac{x_{2}^{2}}{2 \sigma_{z}^{2}}-\frac{\overrightarrow{y^{2}}}{2 \sigma_{x}}\right)
$$

with $\rho_{o}(s-v t)<0$ the linear charge density (the charge per a length unit of accelerator), and

$$
\sigma_{x}=\sqrt{\epsilon_{x} \beta_{x}+\eta^{2} \widetilde{\delta}^{2}}, \sigma_{y}=\sqrt{\epsilon_{y} \beta_{y}}
$$

The solution of Eq.(A.8) is well known ${ }^{[s]}$. We define the value of $U_{o}$ by the following condition

$$
\mathrm{VI}\left(. \quad=\mathrm{a}, \mathrm{y}=0, \mathrm{~s}-v t, \overline{\mathrm{s}}=\overline{\mathrm{s}}_{\mathrm{o}}\right)=0
$$

with $a$, the beam pipe radius in the horizontal plane. The potential $U_{1}$ is given by

$$
U_{1}=-\rho_{o}(s-v t)\left(\int_{0}^{\infty} \frac{e^{-\frac{x^{2}}{2 \sigma_{x}^{2}+q}-\frac{y^{2}}{2 \sigma_{y}^{2}+q}}}{\sqrt{2 \sigma_{x}^{2}+q} \sqrt{2 \sigma_{y}^{2}+q}} d q-\int_{0}^{\infty} \frac{e^{-\frac{a_{o}^{2}}{2 \sigma_{x o}^{2}+q}}}{\sqrt{2 \sigma_{x o}^{2}+q} \sqrt{2 \sigma_{y o}^{2}+q}} d q\right) .
$$

The expansion of these expressions to lowest order in the transverse coordinates $\frac{x^{2}}{2 \sigma_{x}^{2}}$, $\frac{y^{2}}{2 \sigma_{y}^{2}}$ gives the electric field of the bunch near the beam centerline

$$
\begin{aligned}
& E,=-\rho_{o}(s-v t) \frac{2 \mathrm{x}}{\sigma_{x}\left(\sigma_{x}+\sigma_{y}\right)} \\
& E_{y}=-\rho_{o}(s-v t) \frac{2 y}{\sigma_{y}\left(\sigma_{x}+\sigma_{y}\right)} \\
& E_{s}=-2 \rho_{o}(s-v t)\left(\frac{\sigma_{x}^{\prime}+\sigma_{y}^{\prime}}{\sigma_{x}+\sigma_{y}}\right)
\end{aligned}
$$

Now let us find the electric field generated by the potential $U_{2}$. According to the definition (A.16), the boundary condition for the potential $U_{2}$ is

$$
\left.U_{2}\right|_{w a l l}=-\left.U_{1}\right|_{w a l l}
$$

The typical horizontal a, and vertical $a_{y}$ dimensions of the beam pipe satisfy the following conditions : $a, \gg \sigma_{x}, a_{y} \gg \sigma_{y}$. Hence the integral (A.12) may be simplified for 
the region $x^{2} / \sigma_{2}^{2}, y 2 / \sigma_{y}^{2} \gg 1$. The result is

$$
\left.U_{2}\right|_{w a l l} \approx-\rho_{o}(s-v t) \ln \left(\frac{x_{w}^{2}+y_{w}^{2}}{a_{o}^{2}}\right)
$$

where $x_{w}$ and $y_{w}$ parametrize the shape of the wall of the vacuum chamber.

The solution of the Eq. (A.8) inside of the vacuum chamber with the boundary condition Eq. (A.9) depends only on $s-v t$ and of the order of the potential at the boundary. The longitudinal electric field generated by the potential $U_{2}$ is caused by the variation of the beam pipe cross section along the ring and is of the order of

$$
\tilde{E}_{s} \approx 2 \rho_{o} \frac{a^{\prime}}{a}
$$

with a the typical value of the beam pipe aperture. The sign' means the derivative with respect to $\tilde{s}$. It may be important in the process of longitudinal ion motion and in defining the location of the trapped ion pockets along the accelerator because the variations of $a(s)$ occurs at small distances compared with the variations of $\sigma(s)$. These effects for the High Energy Ring will be considered in Chapter 5.

The typical period of ion oscillations in the potential well of the electron beam is much larger than the bunch spacing. Hence, ions see only the average field of a beam. In this approximation, the density $\rho_{0}(s-v t)$ in the Eqs. (A.12), (A.13)-(A.15), should be replaced by the ratio of the charge of a bunch to the bunch spacing, $e N_{B} / s_{B}$.

Appendix B. Simulation of the nonlinear motion of ions.

Here we describe the algorithm of simulation of the nonlinear motion of ions in the average field of the beam with a gap in the train of bunches. The motion is, obviously, is the nonlinear stable oscillations between passage of a gap and free motion within the gap. We consider only the motion with amplitudes small compared to the rms beam size trying to understand how different is result compared to that given by the linear theory. Expansion of the average field of the train in transverse coordinates gives the equation

$$
\frac{d^{2} x}{d t 2}+\Omega_{x}^{2} x=\alpha_{x} x y^{2}+\beta_{x} x^{3}
$$

where

$$
\Omega_{x, y}^{2}=\frac{\Omega_{0}^{2}}{A} \frac{\sigma_{x}+\sigma_{y}}{\sigma_{x, y}}, \quad \Omega_{0}^{2}=\frac{e I Z_{0}}{2 \pi m_{p}\left(\sigma_{x}+\sigma_{y}\right)^{2}}
$$

and

$$
\begin{gathered}
\alpha_{x}=\frac{\Omega_{0}^{2}}{2 A} \frac{1}{\sigma_{x} \sigma_{y}} \\
\beta_{x}=\frac{\Omega_{0}^{2}}{2 A} \frac{\sigma_{y}+2 \sigma_{x}}{3 \sigma_{x}^{3}}
\end{gathered}
$$

Consider, for simplicity, motion only in x-plane. The Hamiltonian in this case is the 
Hamiltonian of the long time-independent octupole:

$$
H=\frac{p^{2}}{2}+\frac{\Omega^{2} x^{2}}{2}-\frac{\beta x^{4}}{4}
$$

To describe such a motion we transform the Hamiltonian to the action- angle canonical variables $J, \phi$ :

$$
\begin{gathered}
x=\sqrt{\frac{2 J}{\Omega}} \cos \phi, \quad p=-\sqrt{2 J \Omega} \sin \phi \\
H=\Omega J-\frac{\beta}{4}\left(\frac{2 J}{\Omega}\right)^{2} \cos ^{4} \phi .
\end{gathered}
$$

Now we can to make the canonical transformation to the new variables $I, \xi$ with a generating function

$$
\begin{gathered}
F(\phi, I)=I \phi+\sum_{n} \int^{\phi} d \psi \lambda_{n}(\psi, I), \\
J=I+\sum_{n} \lambda_{n}(\phi, I), \quad \xi=\phi+\sum_{n} \int^{\phi} d \psi \frac{\partial \lambda_{n}(\psi, I)}{\partial I} .
\end{gathered}
$$

The new Hamiltonian is simply

$$
H(I, \xi)=\mathrm{RI}, \quad I=\text { const }, \quad \xi=\xi(0)+\Omega t
$$

if $\lambda_{n}$ are

$$
\lambda_{n}(\phi, I)=\frac{\beta}{(2 \Omega)^{3}}[3+4 \cos 2 \phi+\cos 4 \phi]\left[I^{2}+2 I \sum_{n=2} \lambda_{n}+\left(\sum \lambda_{n}\right)^{2}\right]
$$

For small amplitudes a of oscillations, the parameter

$$
\frac{6 \beta I}{(2 \Omega)^{3}} \simeq\left(\frac{a}{4 \sigma_{y}}\right)^{2}
$$

is small and $\lambda_{n}$ can be found by expansion over this parameter. That gives

$$
\lambda_{2}=\frac{\beta I^{2}}{(2 \Omega)^{3}}[3+4 \cos 2 \phi+\cos 4 \phi],
$$




$$
\mathrm{A} 3=\frac{2 \beta^{2} I^{3}}{(2 \Omega)^{6}}[3+4 \cos 2 \phi+\cos 4 \phi]^{2} .
$$

Hence, with the accuracy $o\left(\beta^{3}\right)$,

$$
J=I+\lambda_{2}(\phi, I)+\lambda_{3}(\phi, I)
$$

and

$$
\begin{gathered}
\xi=\phi+\frac{2 \beta I}{(2 \Omega)^{3}}\left[3 \phi+2 \sin 2 \phi+\frac{1}{4} \sin 4 \phi\right]+\frac{6 \beta^{2} I^{2}}{(2 \Omega)^{6}} \\
{\left[\frac{35}{2} \phi+14 \sin 24+\frac{7}{2} \sin 44+\frac{2}{3} \sin 64+\frac{1}{16} \sin 8 \phi\right] .}
\end{gathered}
$$

Note, that neglecting all $\lambda_{n}$ with $\mathrm{n} \geq \mathbf{3}$ we obtain approximate integrable Hamiltonian $H=H(I)$ but variables $I, \xi$ are canonical variables exactly, that is the transform from $J, \phi$ to $I, \xi$ is simplectic.

With the same accuracy, the inverse formulas are

$$
I=J-\frac{\beta J^{2}}{(2 \Omega)^{3}}[3+4 \cos 2 \phi+\cos 4 \phi]
$$

and

$$
\begin{gathered}
\phi=\xi-\frac{2 \beta I}{(2 \Omega)^{3}} \Phi\left(\xi-\frac{2 \beta I}{(2 \Omega)^{3}} \Phi(\xi)\right)-\frac{6 \beta^{2} I^{2}}{(2 \Omega)^{6}} \\
{\left[\frac{35}{2} \xi+14 \sin 2 \xi+\frac{7}{2} \sin 4 \xi+\frac{7}{3} \sin 6 \xi+\frac{7}{3} \sin 8 \xi\right]} \\
\frac{16}{3}
\end{gathered}
$$

where

$$
\Phi(\phi)=3 \phi+2 \sin 2 \phi+\frac{1}{4} \sin 4 \phi .
$$

The map for a long octupole can be obtained by going through the following steps:

a)initial $x(0), p(0)$ at the beginning of the train define $J_{0}, \phi_{0}$ :

$$
J_{0}=\frac{p(0)^{2}}{2 \Omega}+\frac{\Omega^{2} x^{2}(0)}{2}, \quad \tan \phi_{0}=-\frac{p(0)}{\Omega x(0)}
$$

b) $J_{0}$, do define $I_{0}\left(J_{0}, \phi_{0}\right)$ and $\xi_{0}\left(\phi_{0}, I o\right)$ using Eqs. (B.6) and (B.5).

c) That gives $I=I_{0}$ and $\xi=\xi_{0}+\Omega\left(T-T_{g}\right)$ at the end of the train.

d) $I, \xi$ define $\phi(I, \xi)$ and $J(I, \phi)$ using Eqs. (B.7) and (B.4). 
e)Finally, $J, \phi$ define $x, p$ at the end of train by Eqs. (B i ) -

Transform through the gap is trivial: $x_{f}=x+p T_{g} / m, p_{f}=p$.

The map obtained in this way is simplectic only with the accuracy of the order of $(a / 4 \sigma)^{6}$. This accuracy is quite acceptable for tracking through 100 turn for the amplitudes $100(a / 4 \sigma)^{4}<<1$ or $a / \sigma<<1$.

\section{REFERENCES}

1. Foster F.Rieke and William Prepejchal,"Ionization Cross sections of Gaseous Atoms and Molecules for High-Energy Electrons and Positrons",Phys. Rev. A,Vol.6,No. 4,p.1507-1519,(1972)

2. L. D. Landau and E. M. Lifshitz, "Quantum Mechanics: Non-Relativistic theory”,Pergamon Press,(1981)

3. PEP-2 Update Design,(Feb.1992)

4. Donald H.Perkins,"Introduction to High Energy Physics", Addison-Wesley Publishing Company,(1972)

5. S. Kheifets, "Potential of a three-dimensional Gauss bunch” , PETRA Note 119 (1.10.76).

6. M. Abramowitz and I.A. Stegun, "Handbook of Mathematical Functions”, National Bureau of Standards, Washington, 1966, formula 7.1.3

7. M. Bassetti and G.A. Erskine "Closed Expression for electrical field of a twodimensional Gaussian Charge”, Geneva, 1980

8. D.Sagan "Some aspects of the longitudinal motion of ions in electron storage rings." Nuclear Instruments and Methods in Physics Research, A 307 (1991), 171-178

9. Y.Miyahara, K.Takayama, G. Horikoshi, "Dynamical analysis on the longitudinal motion of trapped ions in an electron storage ring”. Nuclear Instruments and Methods in Physics Research A 270 (1988) 217-225

10. S. Heifets "Effect of the ion trapping at CEBAF"

11. Fl. Pedersen "RF Cavity feedback" Proceeding of "B-factories, The State of the Art in Accelerators, detectors and Physics",SLAC,April 6-10,1992

12. L. D. Landau and E. M. Lifshitz "Mechanics",Pergamon Press, (1981)

13. M. Abramowitz and I.A. Stegun, "Handbook of Mathematical Functions", National Bureau of Standards, Washington, 1966, formula 7.1.1

14. V.N. Litvinenko, E.A.Perevedentsev "Raschet parametrov puchka v nakopiteliah so sviaziu kolebanii", Proceedings of 6th All Union Conference on Charged Particles Accelerators, Dubna, USSR, v.2, p.285

15. P.F.Tavares "Transverse Distribution of Ions Trapped in an Electron Beam”, CERN-PS/92-55(LP), September 1992

16. D.V.Pestrikov “Collective Issues Related To B-Factories”, KEK Report 92-21, February 1993

17. R.D.Kohaupt "Ion Clearing Mechanism in The Electron-Positron Storage Ring Doris” DESY M1-71-2 (1971) 


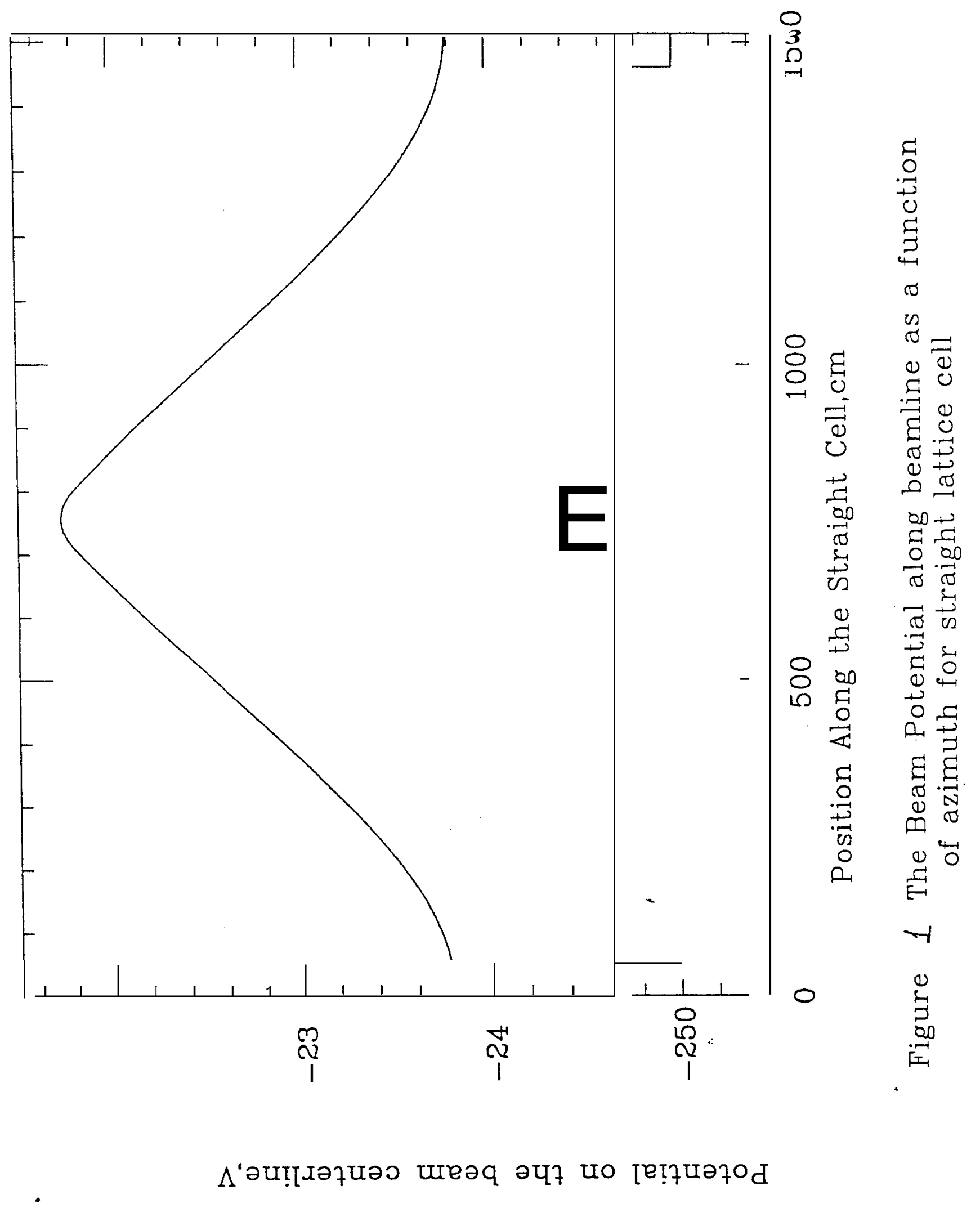




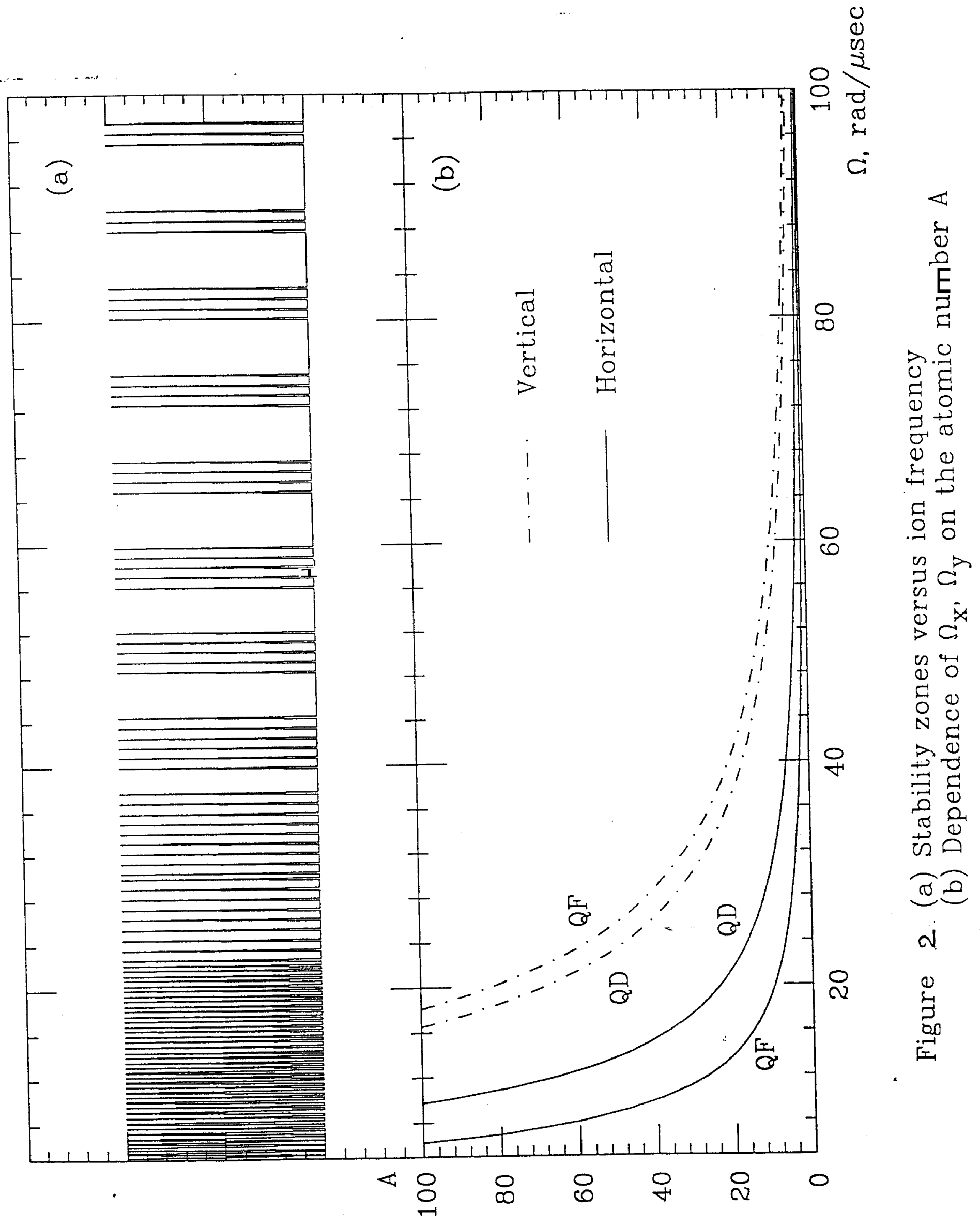




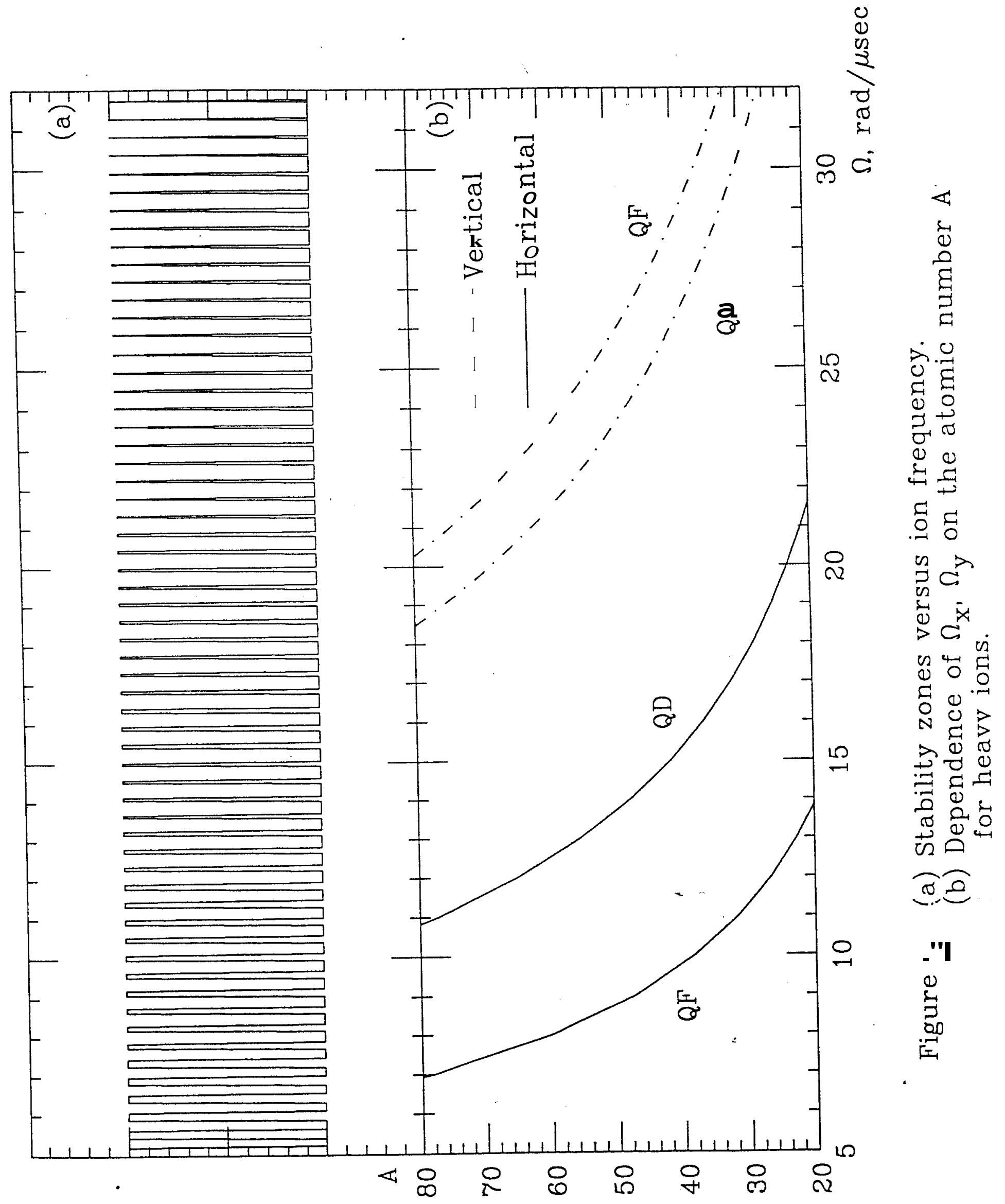




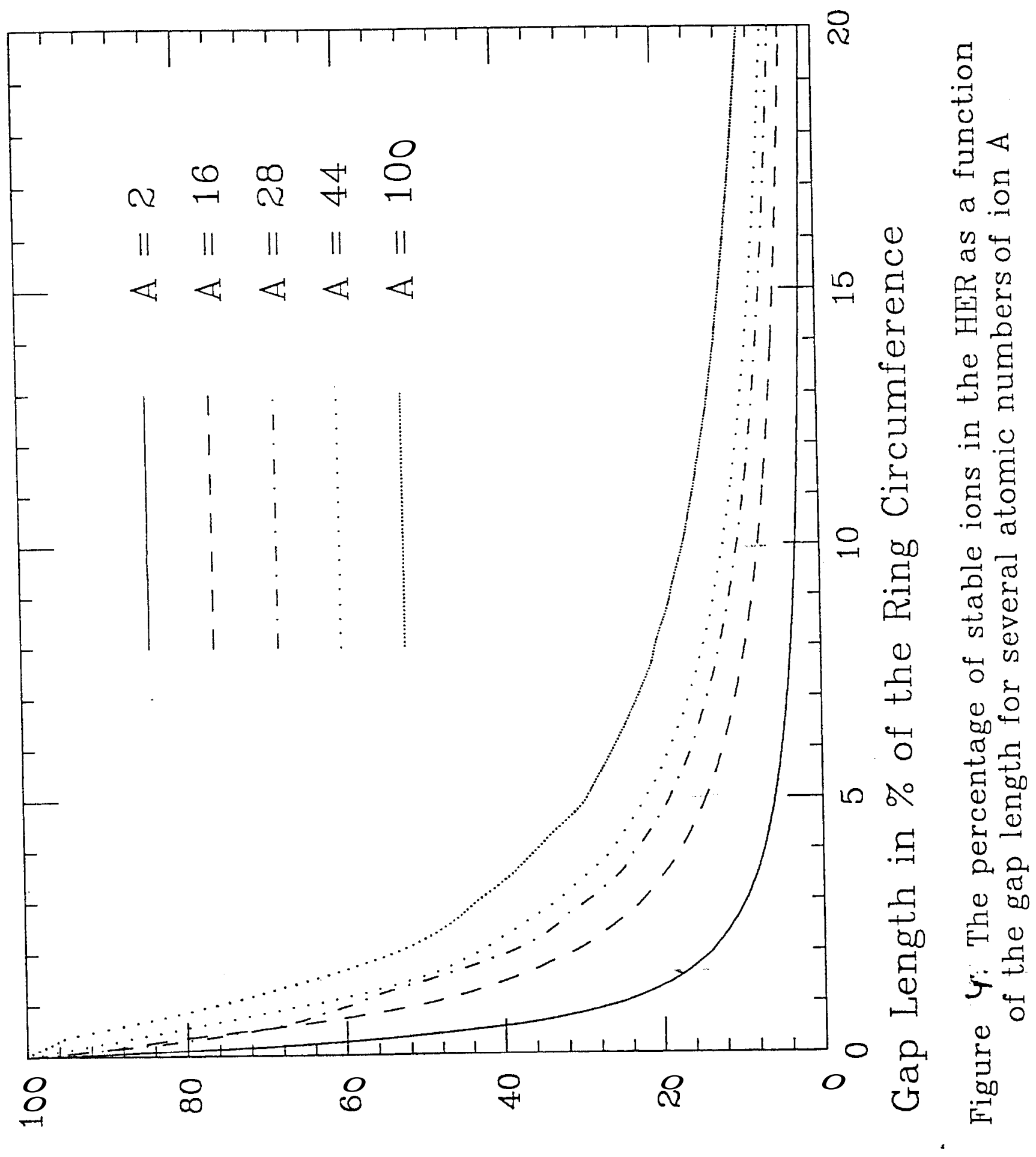

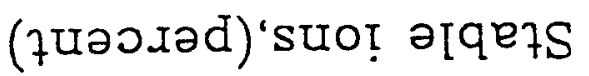




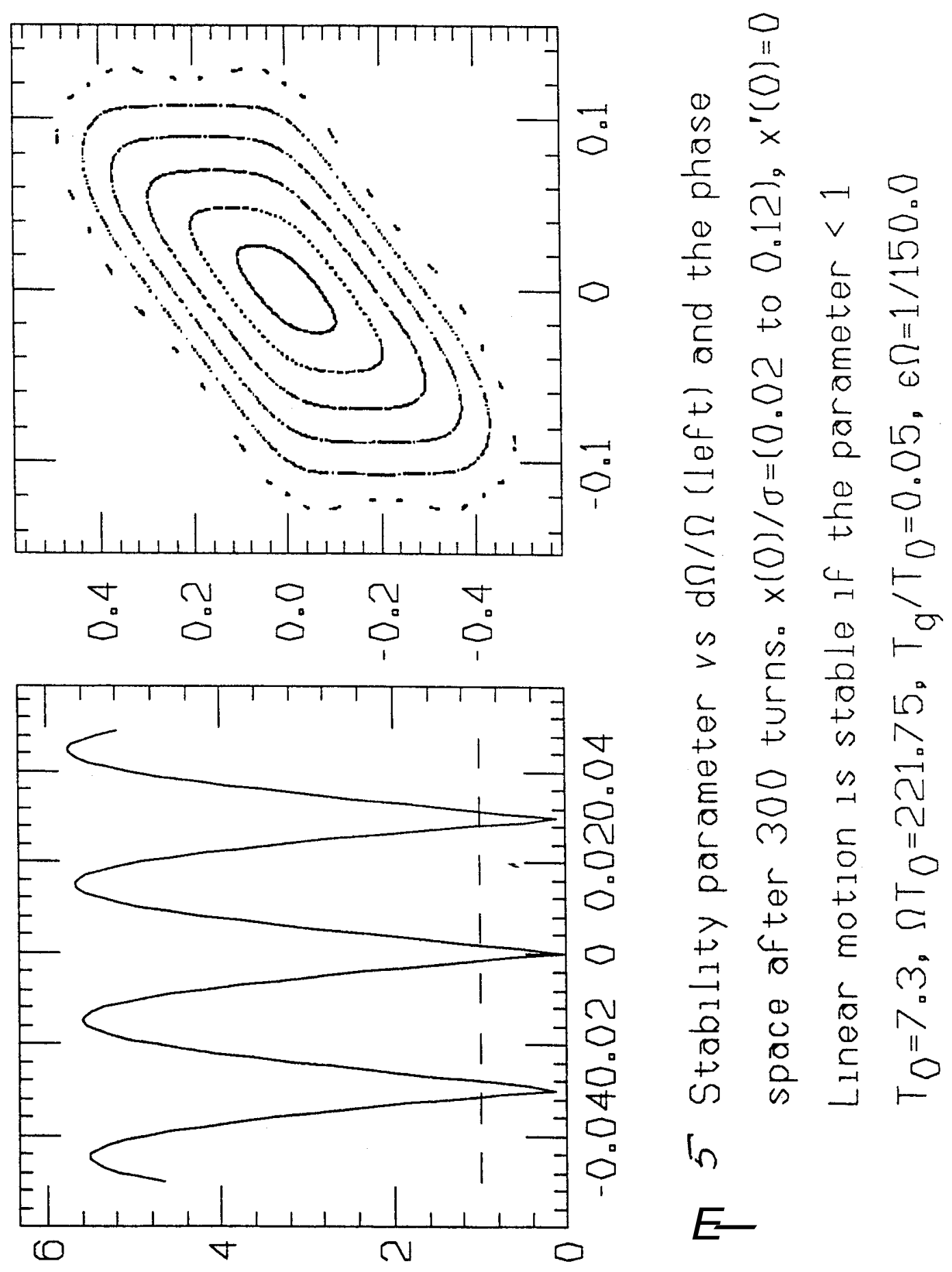




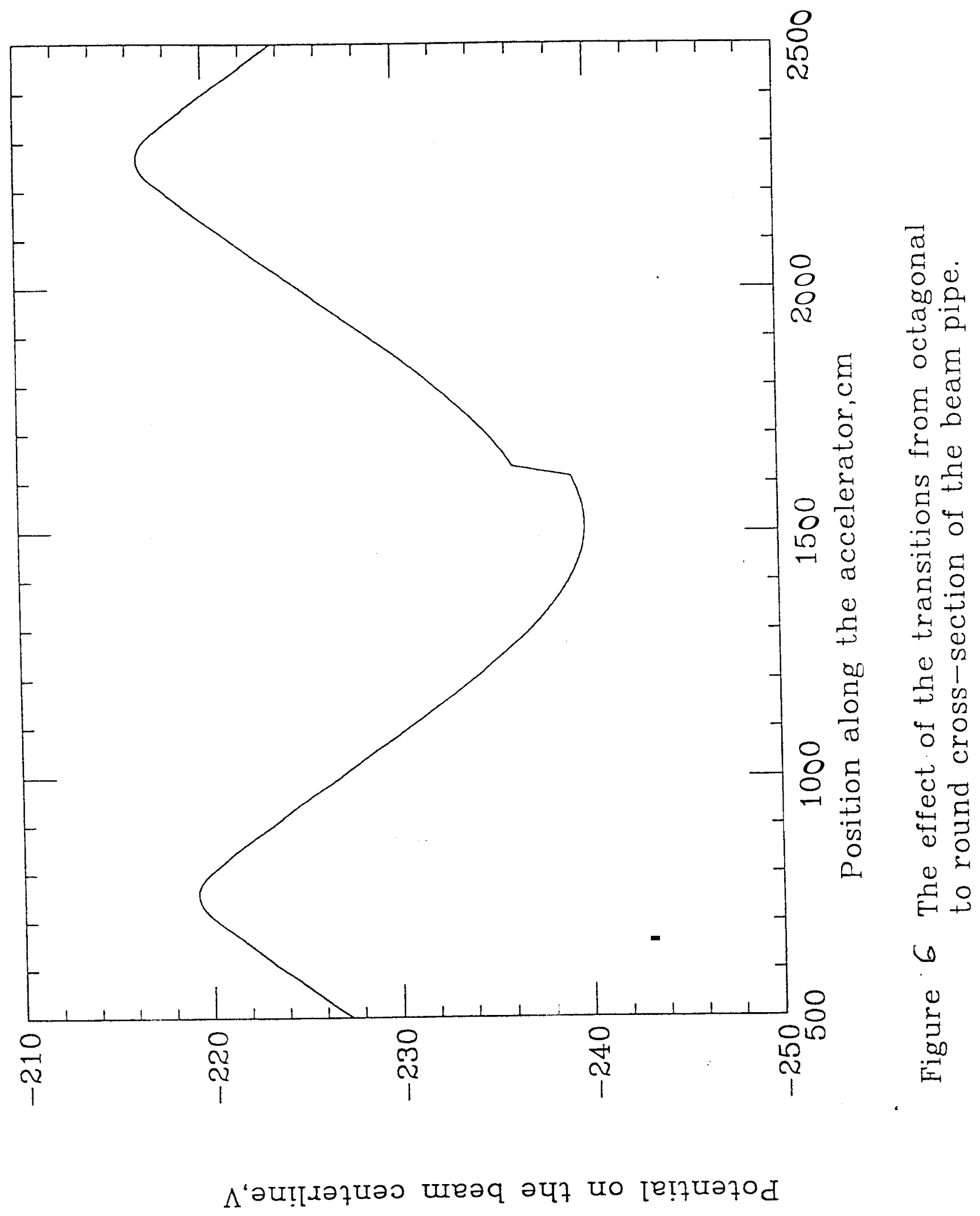




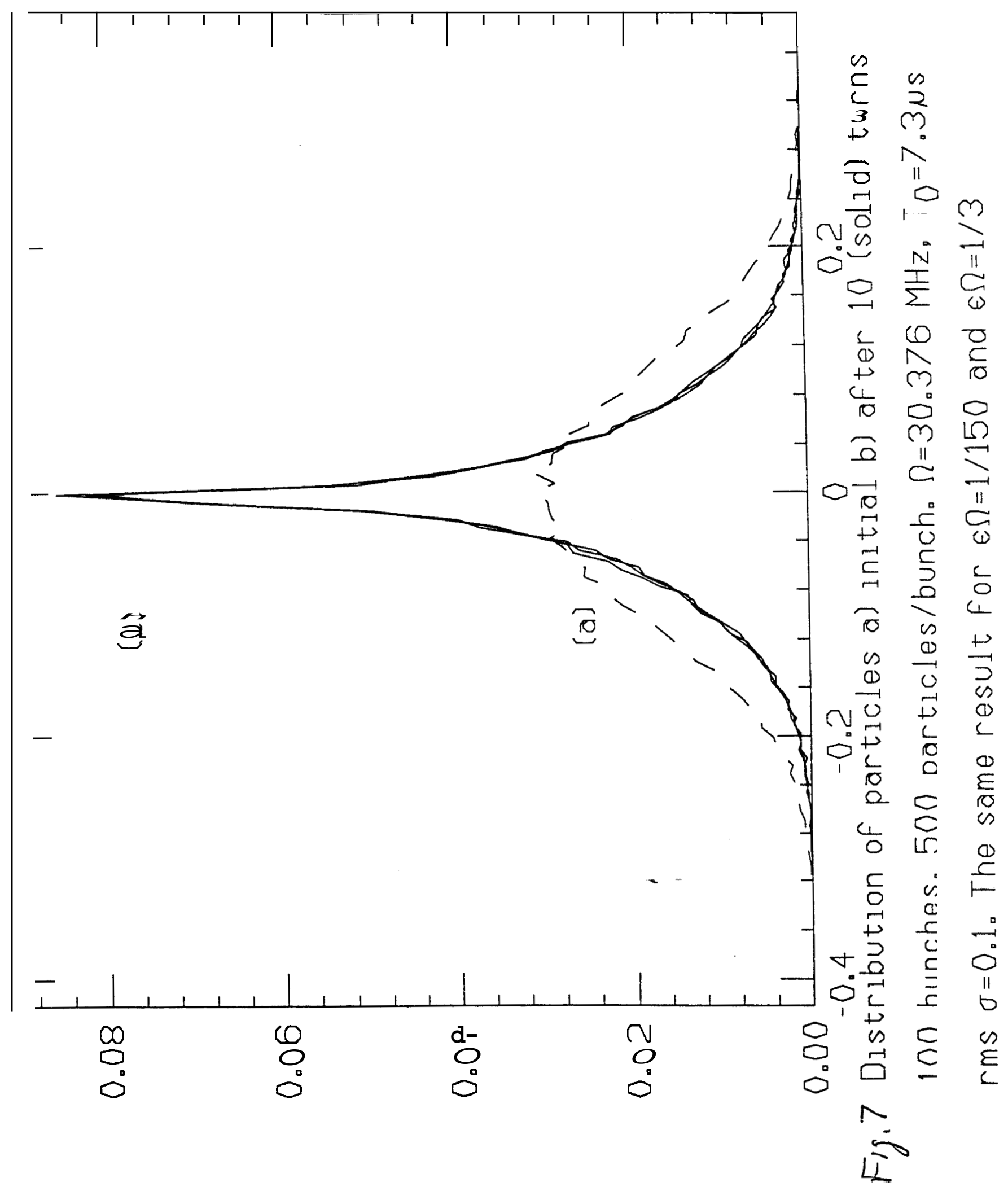




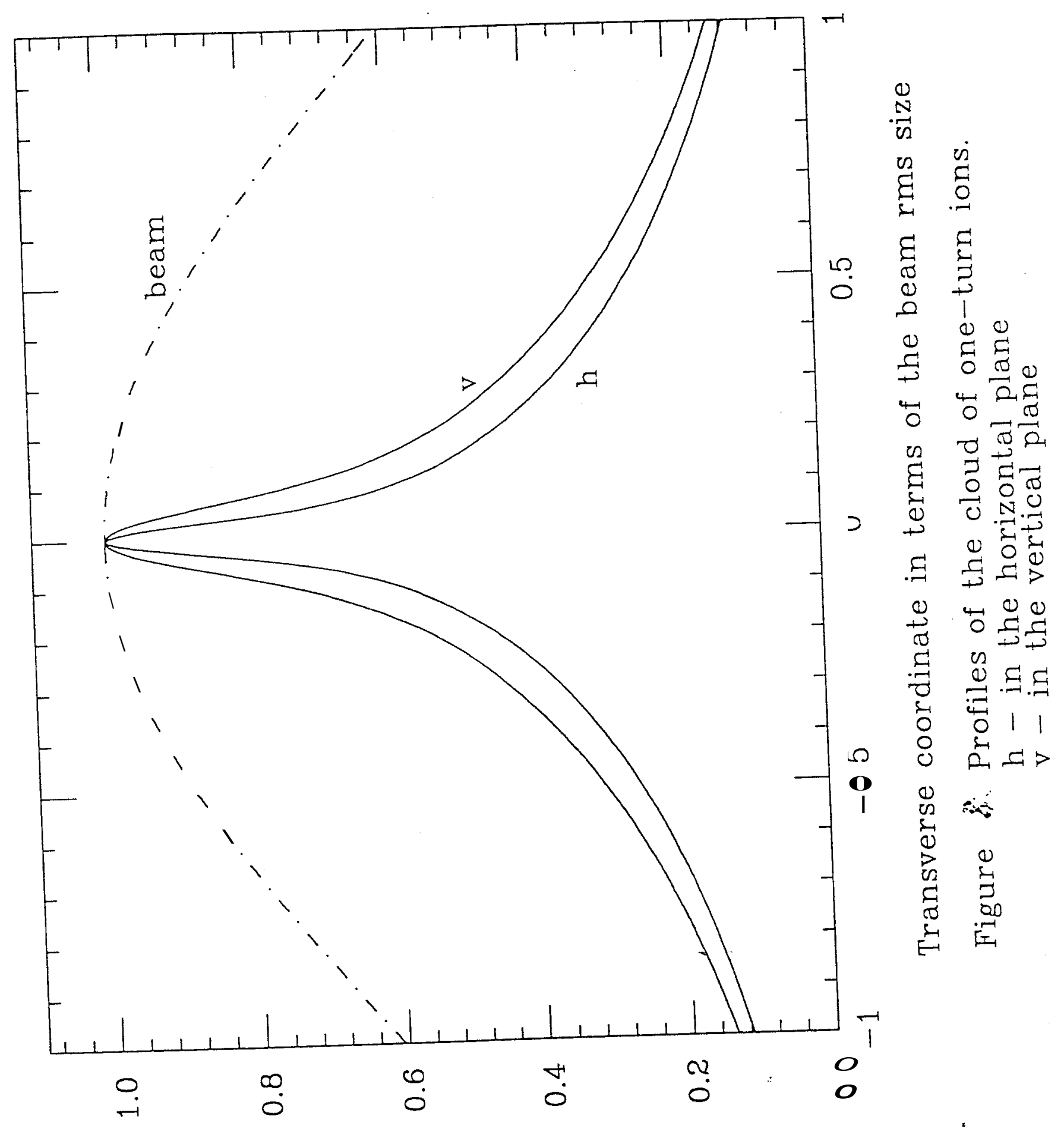

s7tun que'วtyoxd pnojo tol 
Table 3-1. Main PEP-ZZparameters.

\begin{tabular}{|c|c|c|}
\hline & Low-energy fing & High-energy ring \\
\hline Energy, $E[\mathrm{GeV}]$ & 3.1 & 9.0 \\
\hline Circumference, $C[\mathrm{~m}]$ & 2199.32 & 2199.32 \\
\hline Emittance, $\varepsilon_{x} / \varepsilon_{y}[\mathrm{~nm} \cdot \mathrm{rad}]$ & 64.312.6 & $48.2 / 1.9$ \\
\hline Beta function, $\beta_{x}^{*} / \beta_{y}^{*}[\mathrm{~cm}]$ & $37.5 / 1.5$ & 50.012 .0 \\
\hline 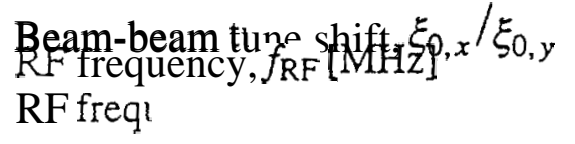 & $\begin{array}{c}0.0310 .03 \\
476\end{array}$ & $\begin{array}{c}0.0310 .03 \\
476\end{array}$ \\
\hline RF voltage, $V_{R F}[M V]$ & 5.9 & 18.5 \\
\hline Bunch length, $\sigma_{2}[\mathrm{~cm}]$ & 1.0 & 1.0 \\
\hline Number of bunches, $k_{B}$ & 1658 & 1658 \\
\hline Bunch separation, $s_{B}[\mathrm{~m}]$ & 1.26 & 1.26 \\
\hline 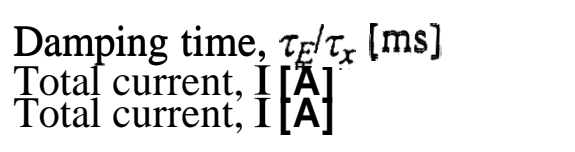 & $\begin{array}{l}19.8 / 40.3 \\
2.14 \\
2.14\end{array}$ & $\begin{array}{c}18.4137 .2 \\
8.99\end{array}$ \\
\hline \$ynch: rad. loss, $U_{0}[\mathrm{MeV} / \mathrm{tum}]$ & $1: 14$ & $3: 58$ \\
\hline Luminosity, $\mathscr{L}\left[\mathrm{cm}^{-2} \mathrm{~s}^{-1}\right]$ & \multicolumn{2}{|c|}{$3 \times 10^{33}$} \\
\hline
\end{tabular}

